\title{
Nonlinear Spectroscopy in the Condensed Phase: The Role of Duschinsky Rotations and Third Order Cumulant Contributions
}

\author{
Tim Zuehlsdorff, Hanbo Hong, Liang Shi, Christine Isborn
}

Submitted date: 14/05/2020 - Posted date: 15/05/2020

Licence: CC BY-NC-ND 4.0

Citation information: Zuehlsdorff, Tim; Hong, Hanbo; Shi, Liang; Isborn, Christine (2020): Nonlinear Spectroscopy in the Condensed Phase: The Role of Duschinsky Rotations and Third Order Cumulant Contributions. ChemRxiv. Preprint. https://doi.org/10.26434/chemrxiv.12302018.v1

First-principles modeling of nonlinear optical spectra in the condensed phase is highly challenging because both environment and vibronic interactions can play a large role in determining spectral shapes and excited state dynamics. Here, we compute two dimensional electronic spectroscopy (2DES) signals based on a cumulant expansion of the energy gap fluctuation operator, with a specific focus on analyzing mode mixing effects introduced by the Duschinsky rotation and the role of the third order term in the cumulant expansion for both model and realistic condensed phase systems. We show that for a harmonic model system, the third order cumulant correction captures effects introduced by a mismatch in curvatures of ground and excited state potential energy surfaces, as well as effects of mode mixing. We also demonstrate that 2DES signals can be accurately reconstructed from purely classical correlation functions using quantum correction factors. We then compute nonlinear optical spectra for the Nile red and Methylene blue chromophores in solution, assessing the third order cumulant contribution for realistic systems. We show that the third order cumulant correction is strongly dependent on the treatment of the solvent environment, revealing the interplay between environmental polarization and the electronic-vibrational coupling.

File list (2)

main_manuscript.pdf (3.17 MiB)

view on ChemRxiv - download file 


\title{
Nonlinear spectroscopy in the condensed phase: The role of Duschinsky rotations and third order cumulant contributions
}

\author{
Tim J. Zuehlsdorff, ${ }^{1,}$ a) Hanbo Hong, ${ }^{1}$ Liang Shi, ${ }^{1,}$ b) and Christine M. Isborn ${ }^{1,}$ c) \\ Chemistry and Chemical Biology, University of California Merced, Merced, California 95343, \\ $U S A$
}

(Dated: 13 May 2020)

\begin{abstract}
First-principles modeling of nonlinear optical spectra in the condensed phase is highly challenging because both environment and vibronic interactions can play a large role in determining spectral shapes and excited state dynamics. Here, we compute two dimensional electronic spectroscopy (2DES) signals based on a cumulant expansion of the energy gap fluctuation operator, with a specific focus on analyzing mode mixing effects introduced by the Duschinsky rotation and the role of the third order term in the cumulant expansion for both model and realistic condensed phase systems. We show that for a harmonic model system, the third order cumulant correction captures effects introduced by a mismatch in curvatures of ground and excited state potential energy surfaces, as well as effects of mode mixing. We also demonstrate that 2DES signals can be accurately reconstructed from purely classical correlation functions using quantum correction factors. We then compute nonlinear optical spectra for the Nile red and Methylene blue chromophores in solution, assessing the third order cumulant contribution for realistic systems. We show that the third order cumulant correction is strongly dependent on the treatment of the solvent environment, revealing the interplay between environmental polarization and the electronic-vibrational coupling.
\end{abstract}

\section{INTRODUCTION}

Excited state dynamics of chromophores in complex environments play an important role in a wide variety of systems, from light harvesting complexes to photoreceptors responsible for vision. ${ }^{1-5}$ Nonlinear optical spectroscopy is capable of probing excited state dynamics on a femtosecond time-scale, revealing ultrafast solventdriven relaxation in organic dyes ${ }^{6-10}$ and efficient energy transfer in multi-chromophore biological systems. ${ }^{11-15}$ However, nonlinear spectroscopy experiments are often difficult to interpret and thus rely heavily on theoretical modeling to connect the observed dynamics to the underlying electronic and atomic motion.

Modeling nonlinear optical experiments in the condensed phase from first principles is highly challenging, as the coupling between electronic and (potentially anharmonic) vibrational degrees of freedom of the chromophore, as well as environmental relaxation and polarization effects, make significant contributions to spectral shapes and spectral evolution. A variety of sophisticated simulation techniques exist that are capable of treating both electronic and nuclear degrees of freedom fully quantum mechanically, ${ }^{16-19}$ but generally have a large computational cost that limits the size of calculations to systems with a few nuclear degrees of freedom. The influence of the complex condensed phase environment then often has to be approximated, for example through classical polarizable continuum models. ${ }^{20-22}$ However, in many situations, such as in energy trans-

\footnotetext{
a) Electronic mail: tzuehlsdorff@ucmerced.edu

b) Electronic mail: lshi4@ucmerced.edu

c) Electronic mail: cisborn@ucmerced.edu
}

fer processes in biological systems, the complex condensed phase environment couples to the excited states of the system to facilitate efficient relaxation. ${ }^{23-27}$ Furthermore, in linear spectroscopy, such as in computing absorption spectra in solvated dyes, a fully atomistic representation of the environment is often necessary to correctly describe specific interactions between the chromophore and its surroundings. ${ }^{28-37}$ To capture polarization effects of the electronic excited state, it is often necessary to treat significant parts of the environment quantum mechanically, ${ }^{28,31,34,35,38-42}$ making efficient electronic structure approaches necessary. ${ }^{43-45} \mathrm{We}$ have recently shown that the electronic-vibrational coupling strength of fast chromophore degrees of freedom to an electronic excitation can also be heavily influenced by the quantum mechanical treatment of the environment. ${ }^{46}$

An appealing way to account for the effect of the complex environment and the coupling of nuclear motion to the optical excitation in linear and nonlinear spectroscopy is the cumulant approach, ${ }^{47}$ where the response function of the system is expressed in terms of a cumulant expansion of the energy gap operator between the electronic ground and excited state. If the energy gap fluctuations obey Gaussian statistics, as they do for harmonic ground and excited state potential energy surfaces (PESs) of the same curvature, then the cumulant expansion is exact at second order. Truncation at second order maps the system dynamics onto a fictitious bath of linearly coupled harmonic oscillators, for which linear and nonlinear spectroscopy signals can be computed analytically. ${ }^{47}$ The electronic-vibrational coupling in the system is then fully defined by the autocorrelation function of energy gap fluctuations, which can be efficiently obtained in complex condensed phase systems by computing vertical excitation energies along molecular dynamics (MD) trajectories ${ }^{23-27,46,48-50}$ and using 
quantum correction factors $(\mathrm{QCF})^{49,51-53}$ to account for nuclear quantum effects.

The second-order cumulant term maps to a harmonic shape for the underlying ground and excited state PESs of the system. For systems with anharmonic degrees of freedom, model calculations demonstrate that higher order cumulants may contribute significantly to both absorption lineshapes ${ }^{54,55}$ and nonlinear spectroscopy signals. ${ }^{56}$ For simulated nonlinear spectra in model systems, the influence of anharmonicity in the PESs can be quite large, especially for long delay times. ${ }^{57} \mathrm{Al}-$ though a harmonic bath model constructed from energy gap fluctuations obtained from MD often forms the basis for studying energy relaxation processes in biological light-harvesting complexes, ${ }^{23-27,49,58-62}$ this relaxation cannot be fully described by a harmonic treatment of the environment. ${ }^{63}$ Even in situations where the chromophore nuclear degrees of freedom can be closely approximated as harmonic, such as in small, rigid systems, the ground- and excited state PESs often have different curvatures and mode mixing within an optical transition, termed a Duschinksy rotation. ${ }^{64}$ Both of these effects introduce non-Gaussian fluctuations to the energy gap and therefore the cumulant expansion truncated at second order is no longer exact. ${ }^{55,56}$ Some of the authors have recently shown that the effect of both Duschinsky mode mixing and changes between the ground- and excited state PES curvature, as well as moderate anharmonicities, on linear absorption spectra can be approximately captured by a cumulant expansion truncated at third order. ${ }^{55}$ The third order cumulant correction can be constructed to good accuracy from a purely classical two-time correlation function of energy gap fluctuations using a recently derived QCF, ${ }^{65}$ opening up the possibility of capturing effects beyond the second order cumulant approximation in complex condensed phase systems.

A harmonic model system termed the generalized Brownian oscillator model (GBOM) has been extensively used in the context of linear spectroscopy ${ }^{66-75}$ The GBOM can include both differing PES curvatures and Duschinsky rotations while still possessing an exact solution for linear spectroscopy (note that a FranckCondon spectrum is exact within the GBOM, assuming the Condon approximation ${ }^{76,77}$ ). Because PES curvature and Duschinsky parameters are obtained by performing a ground and excited state geometry optimization and normal mode analysis using standard electronic structure packages, the GBOM is a convenient model system. A simplified version of the GBOM excluding Duschinsky rotation effects has been used previously ${ }^{56}$ to derive expressions for nonlinear spectroscopy in the third order cumulant approximation.

In this work, we apply the cumulant expansion approach based on classical correlation functions and QCFs to the simulation of nonlinear spectroscopy, specifically two-dimensional electronic spectroscopy (2DES). Using the known classical and quantum correlation functions of energy gap fluctuations for the GBOM, ${ }^{55}$ we derive analytical expressions for the 2DES signal in the third order cumulant approximation that include Duschinsky rotation effects. We use the GBOM to first demonstrate the effect of curvature changes and Duschinsky rotation effects on a simple 2-mode model system, then compute and analyze 2DES signals for the Nile red molecule while isolating the effects of the Duschinsky rotation. To showcase the strengths of the third order cumulant approach based on classical correlation functions, we then compute 2DES signals for the Methylene blue molecule in water both with a GBOM and directly from energy-gap correlation functions calculated from an MD trajectory. For the MD snapshots, we explicitly treat large parts of the solvent environment quantum mechanically to capture the influence of polarization on vertical excitation energies. We show that the third order cumulant correction is particularly sensitive to the treatment of solute-solvent interactions, in agreement with a similar finding for the second order cumulant approach ${ }^{46}$

\section{THEORETICAL BACKGROUND}

\section{A. Linear absorption spectra in the cumulant approach}

For the purpose of this work, we limit the discussion to a two-level electronic system, consisting of an electronic ground state and a single electronic excited state, coupled to nuclear motion. The effective Hamiltonian for this system can be written as

$$
\begin{aligned}
\hat{H} & =|g\rangle H_{g}(\hat{\mathbf{q}})\langle g|+| e\rangle H_{e}(\hat{\mathbf{q}})\langle e|, \\
\hat{V} & =|g\rangle \mu_{g e}(\hat{\mathbf{q}})\langle e|+| e\rangle \mu_{e g}(\hat{\mathbf{q}})\langle g|,
\end{aligned}
$$

where $g$ and $e$ denote the electronic ground and first excited state, respectively, $\hat{\boldsymbol{q}}$ is the operator of nuclear coordinates, $H_{g}(\hat{\mathbf{q}})$ and $H_{e}(\hat{\mathbf{q}})$ are the nuclear Hamiltonians corresponding to the ground and excited electronic states, and $\mu_{e g}(\hat{\mathbf{q}})=\mu_{g e}(\hat{\mathbf{q}})^{\dagger}$ are the transition dipole operators. We assume that the Condon approximation ${ }^{76,77}$ is valid, such that $\mu_{e g}(\hat{\mathbf{q}}) \approx \mu_{e g}$, i.e. the transition dipole operator is independent of nuclear coordinates.

Under these approximations, it is possible to write the linear absorption spectrum (the one-dimensional electronic spectrum, 1DES) of the system in terms of a cumulant expansion of the energy gap fluctuation operator $\delta U(\hat{\boldsymbol{q}})=H_{e}-H_{g}-\omega_{\mathrm{eg}}^{\mathrm{av}}:{ }^{47}$

$$
\begin{aligned}
S_{1 \text { DES }}(\omega) & \propto \operatorname{Re} \int_{0}^{\infty} \mathrm{d} t e^{\mathrm{i}\left(\omega-\omega_{\mathrm{eg}}^{\mathrm{av}}\right) t} \\
& \times\left\langle\exp _{+}\left[-\mathrm{i} \int_{0}^{t} \mathrm{~d} \tau \delta U[\hat{\boldsymbol{q}}(\tau)]\right]\right\rangle \\
& \propto \operatorname{Re} \int_{0}^{\infty} \mathrm{d} t e^{\mathrm{i}\left(\omega-\omega_{\mathrm{eg}}^{\mathrm{av}}\right) t} \exp \left[\sum_{n=2}^{\infty} g_{n}(t)\right] .
\end{aligned}
$$

Here, $\omega_{\mathrm{eg}}^{\mathrm{av}}=\langle U(\hat{\boldsymbol{q}})\rangle$ is the thermal average of the energy gap operator, $g_{n}(t)$ is the $n^{t h}$ order cumulant and 
Hartree atomic units are used throughout. If the energy gap fluctuations are Gaussian, as is true for the quadratic potential of a simple displaced harmonic oscillator system where ground and excited state energy surfaces have the same curvature, the cumulant expansion in Eqn. 2 can be exactly truncated at second order. ${ }^{47}$ For more realistic non-harmonic potential energy surfaces, higher order cumulants will contribute to the lineshape. ${ }^{54,55}$ The cumulants can be expressed in terms of time-ordered integrals of increasing orders of quantum time-correlation functions $C_{\delta U}$ of the energy gap fluctuation operator $\delta U$. For the second and third order cumulant, we can write

$$
\begin{aligned}
g_{2}\left[C_{\delta U}^{\{2\}}\right](t)= & \int_{0}^{t} \mathrm{~d} \tau_{2} \int_{0}^{\tau_{2}} \mathrm{~d} \tau_{1} C_{\delta U}^{\{2\}}\left(\tau_{2}-\tau_{1}\right) \\
g_{3}\left[C_{\delta U}^{\{3\}}\right](t)= & -\mathrm{i} \int_{0}^{t} \mathrm{~d} \tau_{3} \int_{0}^{\tau_{3}} \mathrm{~d} \tau_{2} \times \\
& \int_{0}^{\tau_{2}} \mathrm{~d} \tau_{1} C_{\delta U}^{\{3\}}\left(\tau_{2}-\tau_{1}, \tau_{3}-\tau_{1}\right),
\end{aligned}
$$

where

$$
\begin{aligned}
C_{\delta U}^{\{2\}}(t) & =\langle\delta U(t) \delta U(0)\rangle \\
C_{\delta U}^{\{3\}}\left(t_{1}, t_{2}\right) & =\left\langle\delta U\left(t_{2}\right) \delta U\left(t_{1}\right) \delta U(0)\right\rangle,
\end{aligned}
$$

with analogous expressions for higher order quantum correlation functions. ${ }^{54}$

The second order cumulant contribution $g_{2}(t)$ is commonly expressed in terms of the spectral density of system-bath coupling $\mathcal{J}(\omega)$ by switching into Fourier space and evaluating the time-ordered integrals analytically: ${ }^{47}$

$$
\begin{aligned}
g_{2}(t)= & \frac{1}{\pi} \int_{0}^{\infty} \mathrm{d} \omega \frac{\mathcal{J}(\omega)}{\omega^{2}}\left[\operatorname{coth}\left(\frac{\beta \omega}{2}\right)[1-\cos (\omega t)]\right. \\
& -\mathrm{i}[\sin (\omega t)-\omega t]]
\end{aligned}
$$

with $\beta=1 /\left(k_{\mathrm{B}} T\right)$ and

$$
\mathcal{J}(\omega)=\mathrm{i} \theta(\omega) \int \mathrm{d} t e^{\mathrm{i} \omega t} \operatorname{Im} C_{\delta U}(t) .
$$

A similar expression can be derived ${ }^{55}$ for the third order correction $g_{3}(t)$ (See SI Sec. IB). We have recently demonstrated $^{55}$ that the third order cumulant correction can yield significant improvements in the absorption lineshapes of condensed phase systems with moderately anharmonic nuclear degrees of freedom. In the present work, we extend the analysis of the third order contribution to the simulation of nonlinear electronic spectra.

\section{B. Nonlinear spectroscopy in the cumulant approach}

In this work, we simulate 2DES, which can be related to the third order response function of the system. ${ }^{47} \mathrm{We}$ again limit the discussion to a two-level system consisting of an electronic ground and excited state, meaning that phenomena such as excited state absorption ${ }^{78}$ or nonadiabatic effects due to crossings of excited state potential energy surfaces ${ }^{79}$ are not considered. The third order response function can then be constructed in terms of the four contributions $R_{1}-R_{4}$ that are obtained by considering different double-sided Feynman diagrams. ${ }^{47}$ The total absorptive 2DES signal for a given delay time $t_{\text {delay }}$ is proportional to the sum of the rephasing $\left(R_{2}\right.$ and $\left.R_{3}\right)$ and non-rephasing $\left(R_{1}\right.$ and $\left.R_{4}\right)$ diagrams such that

$$
\begin{array}{r}
S_{2 \mathrm{DES}}\left(\omega_{3}, t_{\text {delay }}, \omega_{1}\right) \propto \operatorname{Re} \int_{0}^{\infty} \mathrm{d} t_{3} \int_{0}^{\infty} \mathrm{d} t_{1} \times \\
{\left[e^{\mathrm{i} \omega_{3} t_{3}+\mathrm{i} \omega_{1} t_{1}}\left(R_{1}\left(t_{3}, t_{\text {delay }}, t_{1}\right)+R_{4}\left(t_{3}, t_{\text {delay }}, t_{1}\right)\right)\right.} \\
\left.e^{-\mathrm{i} \omega_{3} t_{3}+\mathrm{i} \omega_{1} t_{1}}\left(R_{2}\left(t_{3}, t_{\text {delay }}, t_{1}\right)+R_{3}\left(t_{3}, t_{\text {delay }}, t_{1}\right)\right)\right] .
\end{array}
$$

As is the case for linear spectroscopy, the $R_{1}-R_{4}$ terms in the third order response function can be written exactly in terms of the second order cumulant of the energy gap operator if $\delta U$ follows Gaussian statistics. For nonGaussian fluctuations, in principle an infinite number of higher order cumulants contribute to the nonlinear response function. However, Fidler et al. ${ }^{56}$ showed that under the third order cumulant approximation the terms $R_{1}-R_{4}$ can be split into a second order cumulant term and a third order cumulant correction

$$
R\left(t_{3}, t_{\text {delay }}, t_{1}\right) \approx R^{\{2\}}\left(t_{3}, t_{\text {delay }}, t_{1}\right) R^{\{3\}}\left(t_{3}, t_{\text {delay }}, t_{1}\right) .
$$

The second order cumulant contribution $R^{\{2\}}$ can be fully expressed in terms of the function $g_{2}(t)$ (See SI Sec. IA and Ref. 47). In this work, we derive a general expression for the third order cumulant term $R^{\{3\}}\left(t_{3}, t_{\text {delay }}, t_{1}\right)$ that is valid for arbitrary two-time correlation functions $C_{\delta U}^{\{3\}}$ (See SI Sec. IA). $R^{\{3\}}\left(t_{3}, t_{\text {delay }}, t_{1}\right)$ can be written in terms of combinations of time-ordered and non-time-ordered integrals over the two-time correlation function $C_{\delta U}^{\{3\}}$. SI Sec. IB details how to efficiently evaluate the resulting expressions by switching into Fourier space.

For linear spectroscopy, the absorption lineshape in condensed phase systems is dominated by the short timescale behavior of the linear response function, which is often well-approximated by a cumulant approximation truncated at second or third order. ${ }^{55}$ Because 2DES probes longer timescale behavior where the system relaxes on the excited state PES, for these longer delay times the higher order cumulant contributions might become more important or might lead to divergences. ${ }^{54,55}$ However, in the limit of short delay times, the third order cumulant correction will approximately account for non-Gaussian energy gap fluctuations, such as those occurring if the ground- and excited state potential energy surfaces have different curvatures. ${ }^{56}$ In this work, our analysis goes beyond a simple 1D harmonic model system in order to describe the effects of Duschinsky rotations as well as more realistic condensed phase systems. 


\section{Quantum correction factors (QCFs)}

Because the exact quantum correlation functions $C_{\delta U}^{\{n\}}$ are generally inaccessible for anything but the most simple model systems, exactly evaluating cumulant terms in realistic systems is impossible. For this reason, effort has been focused on approximately reconstructing quantum correlation functions from their classical counterparts $C_{\delta U}^{\{n\}, \mathrm{cl}}$ using quantum correction factors (QCFs). ${ }^{51-53}$ Classical correlation functions are generally much easier to compute than their quantum counterparts and can, for example, be constructed by calculating vertical excitation energies along an MD trajectory. ${ }^{23-27,46,48-50}$

Although the choice of QCF is not unique, ${ }^{49,52}$ a commonly used approximation for the energy-gap autocorrelation function $C_{\delta U}^{\{2\}}(t)$ is the harmonic QCF, where

$$
C_{\delta U}^{\{2\}}(\omega) \approx \frac{\beta \omega}{1-e^{-\beta \omega}} C_{\delta U}^{\{2\}, \mathrm{cl}}(\omega)
$$

and $C_{\delta U}^{\{2\}}(\omega)$ is the Fourier representation of $C_{\delta U}^{\{2\}}(t)$ (see SI Sec. IB). The effect of the harmonic QCF is a rescaling of the coupling strength of high frequency vibrational modes to energy gap fluctuations (see SI Eqn. 26) to account for zero-point motion not included in a classical MD trajectory. This harmonic QCF can be derived by establishing a link between the full quantum correlation function and its Kubo-transformed analogue ${ }^{80,81}$ that possesses the same symmetries as the classical correlation function. Recently, a similar expression has been derived for the two-time quantum correlation function $C^{\{3\}}$ through a double-Kubo transform, ${ }^{65}$ where the quantum correlation function can then be approximated via:

$$
C_{\delta U}^{\{3\}}\left(\omega, \omega^{\prime}\right) \approx \frac{\omega \omega^{\prime} \bar{\omega} \beta^{2} C_{\delta U}^{\{3\}, \mathrm{cl}}\left(\omega, \omega^{\prime}\right)}{2\left(\omega^{\prime} e^{-\beta \bar{\omega}}-\bar{\omega} e^{-\beta \omega^{\prime}}+\omega\right)}
$$

where $\bar{\omega}=\omega+\omega^{\prime}$. The QCFs for the one- and two-time correlation functions introduced in Eqns. 11 and 12 have been shown to successfully reproduce linear absorption spectra in the second and third order cumulant approximation for a number of exactly solvable model systems with small to moderately large non-Gaussian energy gap fluctuations. ${ }^{55}$ In the present work we extend this analysis to nonlinear spectroscopy and determine whether QCFs can be used to accurately construct 2DES signals from classical input only.

\section{THE GENERALIZED BROWNIAN OSCILLATOR MODEL (GBOM)}

To investigate the influence of non-Gaussian energy gap fluctuations on computed 2D electronic spectra, we turn to the GBOM Hamiltonian that has been used in a previous study in the context of linear spectroscopy. ${ }^{55}$ In this model, the ground and excited state PESs are harmonic, but their curvatures can differ, and the ground state normal mode coordinates can be rotated with respect to the excited state normal mode coordinates, termed a Duschinsky rotation. ${ }^{64}$

The GBOM is an appealing model system for a number of reasons. First, a Hamiltonian of this form can be parametrized from ground and excited state geometry optimization and normal mode calculations and can thus be readily constructed for a wide variety of systems using standard electronic structure packages. Furthermore, for this simplified Hamiltonian form, exact analytical expressions for the linear response function are known, ${ }^{73,75}$ which some of the authors have recently used to assess the effect of truncating the cumulant expansion at third order in the context of linear spectroscopy. ${ }^{55}$ Although general closed-form expressions for nonlinear spectra for the GBOM are not available, comparing how well the first two cumulants $g_{2}(t)$ and $g_{3}(t)$ approximate the exact linear absorption spectrum could potentially be used as a guide for how well $g_{2}(t)$ and $g_{3}(t)$ approximate the nonlinear response function for short delay times. Finally, the simple form of the Hamiltonians in the GBOM means that analytic expressions for the exact one- and two time correlation functions $C_{\delta U}^{\{2\}}$ and $C_{\delta U}^{\{3\}}$, as well as their classical counterparts, can be determined (Ref. 55, see also SI Sec. IIA and B), allowing for a detailed comparison of the errors introduced into nonlinear spectroscopy calculations when approximately reconstructing the quantum correlation function from purely classical input.

In the GBOM, the nuclear Hamiltonians for the electronic ground and excited state with $N_{j}$ vibrational modes can be written as:

$$
\begin{aligned}
& H_{g}\left(\hat{\mathbf{q}}_{g}, \hat{\mathbf{p}}_{g}\right)=\frac{1}{2} \sum_{j}^{N_{j}}\left[\hat{p}_{g, j}^{2}+\omega_{g, j}^{2} \hat{q}_{g, j}^{2}\right], \\
& H_{e}\left(\hat{\mathbf{q}}_{e}, \hat{\mathbf{p}}_{e}\right)=\frac{1}{2} \sum_{j}^{N_{j}}\left[\hat{p}_{e, j}^{2}+\omega_{e, j}^{2} \hat{q}_{e, j}^{2}\right]+\Delta_{\mathrm{eg}}^{0},
\end{aligned}
$$

where the ground and excited state normal modes are related through the linear transformation:

$$
\hat{q}_{g, i}=\sum_{j}^{N_{j}} J_{i j} \hat{q}_{e, j}+K_{i} .
$$

Here, $K_{i}$ is the shift vector describing the displacement between ground and excited state PES minima, $\Delta_{\text {eg }}^{0}$ is the adiabatic energy gap between the surfaces, and $\mathbf{J}$ is the Duschinsky rotation matrix. Both the difference in curvature, with $\omega_{g, i} \neq \omega_{e, i}$, and the Duschinsky rotation lead to a nonlinear dependence on nuclear coordinates of the operator $\delta U\left(\hat{\mathbf{q}}_{g}\right)$ and thus non-Gaussian energy gap fluctuations (see SI Sec. IIA and Ref. 55), meaning that the second order cumulant approximation is no longer exact. Note that if $\mathbf{J}=\mathbf{I}$, where $\mathbf{I}$ is the identity matrix, all normal modes are completely decoupled. In this simplified case, the only contribution to the non-Gaussian fluc- 
tuations in the GBOM Hamiltonian is from the changes in PES curvature.

To add a model for the condensed phase environment, we follow the same approach as discussed in Ref. 55 to describe the bath as an infinite set of displaced harmonic oscillators. In this GBOM plus solvent model, the chromophore degrees of freedom described by the GBOM contain nonlinear contributions to the energy-gap fluctuations and the condensed phase environment is described by a spectral density of the Debye form:

$$
\mathcal{J}_{\text {solv }}(\omega)=2 \lambda_{\text {solv }} \omega_{c} \frac{\omega}{\omega_{c}^{2}+\omega^{2}},
$$

where $\omega_{c}$ is a characteristic cutoff freqeuncy describing the time-scale of environment relaxation and $\lambda_{\text {solv }}$ is the system-dependent solvent reorganization energy describing the amount of solvent broadening introduced in the spectra. Because the solvent environment is described in terms of a bath of linearly coupled displaced harmonic oscillators, it only contributes to the second order cumulant terms, whereas the third order cumulant corrections are due to the nonlinearly coupled "chromophore" degrees of freedom described by the GBOM. For all GBOM-based calculations reported in this work, the phenomenological cutoff frequency is set to $\omega_{c}=22 \mathrm{~cm}^{-1}$, such that the time-scale of solvent relaxation is much slower than the "chromophore" modes described by the GBOM.

Although the harmonic GBOM has formed the basis of a wide range of linear spectroscopy studies,${ }^{70,73,74,82-86}$ it has substantial limitations. For semi-flexible systems, large-amplitude geometry changes can occur between the ground- and excited state optimized geometry, often leading to a breakdown of the harmonic approximation for the shape of the PESs. Several techniques exist to address this problem, such as constructing GBOMs for high frequency harmonic modes only and treating low frequency anharmonic modes classically, ${ }^{83}$ or by making use of curvilinear coordinates better suited to describing large amplitude motion. ${ }^{86,87}$ Furthermore, the GBOM described in this work is unable to capture specific interactions between the chromophore and its complex environment, such as hydrogen bonding. For this reason, a GBOM likely only forms a good description of the nuclear Hamiltonian for relatively small, rigid chromophores in weakly interacting solvent environments.

\section{COMPUTATIONAL DETAILS}

To asses the role of the third order cumulant approximation as applied to nonlinear spectroscopy, as well as to test the errors introduced when constructing the required quantities from purely classical input, we focus on three systems of increasing complexity. First, we consider a two-mode model system of the GBOM form, the smallest system that allows us to explore the effect of nonGaussian energy gap fluctuations introduced through both the change in ground and excited state curvature and the Duschinsky rotation. We then focus on a more realistic multi-mode system described by the GBOM by calculating ground and excited state normal modes and frequencies for the Nile red molecule in an implicit solvent model for benzene. Finally, to go beyond the GBOM, we focus on the Methylene blue molecule in water where we compare parameterizing a GBOM from electronic structure calculations to results obtained from constructing the classical correlation functions directly from sampling the vertical excitation energy along MD trajectories of the solvated system. Unlike the GBOM-based approach, this latter approach captures anharmonicities and both direct and indirect chromophore-solvent interactions.

For the two-mode GBOM system, we use the same parameters for ground and excited state frequencies, as well as shift vector $\mathbf{K}$ as discussed in Ref. 55 . The valules are $\omega_{g, 1}=834 \mathrm{~cm}^{-1}$ and $\omega_{g, 2}=1251 \mathrm{~cm}^{-1}$, with corresponding excited state frequencies of $\omega_{e, 1}=876 \mathrm{~cm}^{-1}$ and $\omega_{e, 2}=1189 \mathrm{~cm}^{-1}$. In the two-mode model system, the Duschinsky matrix is simply given by the $2 \mathrm{D}$ rotation matrix $\mathbf{R}(\phi)$ and we consider a rotation angle of $\phi=10^{\circ}$. This parameter choice is representative of the amount of mode mixing and change in frequency between ground and excited state normal modes typically encountered in realistic molecular systems.

The geometry optimizations and normal mode calculations necessary to parameterize a GBOM for Nile red and Methylene blue were performed using the CAMB3LYP $/ 6-31+\mathrm{G}^{* 88,89}$ level of theory as implemented in the Gaussian electronic structure package.$^{90}$ Excited state geometry optimizations were performed using timedependent density-functional theory (TDDFT) within the Tamm-Dancoff approximation. ${ }^{91}$ For Nile red the geometry optimizations included the effect of the non-polar solvent benzene by making use of a polarizable continuum model (PCM). ${ }^{20,92}$ For Methylene blue, a PCM model of water leads to divergences in the linear response functions in the third order cumulant approximation, suggesting large non-Gaussian contributions to the energy gap fluctuations, which can likely be traced to large changes in geometry between the ground- and excited state minimum for a few select normal modes. For this reason, the geometry optimizations and normal mode calculations for the Methylene blue GBOM results reported in the main text were instead obtained in vacuum (See SI Secs. IVA and VB for additional computational details of the parameterization of the GBOM for Nile red and Methylene blue).

For Methylene blue, we also directly construct classical correlation functions from $\mathrm{MD}$, thus sampling the full (potentially anharmonic) PES. Six independent 8 ps trajectories using mixed quantum mechanical/molecular mechanical (QM/MM) dynamics on the ground state potential energy surface were generated using the Terachem code, ${ }^{43,93}$ treating the chromophore at the CAM-B3LYP $/ 6-31+\mathrm{G}^{*}$ level of theory and the water molecules with the TIP3P ${ }^{94}$ model. Treating the chromophore degrees of freedom at the CAM-B3LYP/6- 
$31+\mathrm{G}^{*}$ level during the dynamics is crucial to avoid well-known artifacts in the computed energy gap correlation functions resulting from a mismatch between Hamiltonians. ${ }^{48,60,95-98}$ Snapshots are extracted every $2 \mathrm{fs}$, and TDDFT vertical excitation energies are computed using the Tamm-Dancoff approximation, where all solvent molecules within $6 \AA$ of the chromophore are treated fully quantum mechanically to capture polarization effects from first principles. ${ }^{28,31,34,35,38,39,41,42,46}$ All other surrounding water molecules are included in the excited state calculation as classical point charges. Although this QM treatment of the solvent when computing the vertical excitation energies leads to a small Hamiltonian mismatch between dynamics and excited state calculations, we have found in previous studies that this polarization can rather dramatically affect the spectral densities and we choose here to include the environment at the QM level. Classical one- and two-time correlation functions $C_{\delta U}^{\{2\}, \mathrm{cl}}$ and $C_{\delta U}^{\{3\}, \mathrm{cl}}$ are constructed from the total data set of 24,000 TDDFT excitation energies. Further computational details for the MD calculations can be found in SI Sec. V.A.

\section{RESULTS AND DISCUSSION}

\section{A. A 2-mode model system}

The linear absorption spectra for the two-mode GBOM are shown in Fig. 1a, with the exact spectrum shown in gray, compared to that computed with quantum correlation functions within the second and third order cumulant approximations (green and blue lines), and that computed from purely classical correlation functions combined with the QCFs introduced in Sec. II C (yellow and red lines). For the second order cumulant approximation, the QCF results are indistinguishable from the spectrum computed using the exact quantum correlation function (the yellow line is on top of the green line). This agreement is because for this specific harmonic model system, the harmonic QCF exactly reproduces the dominant part of the quantum correlation function (See Ref. 55 and SI Sec. IIIA). For the third order cumulant approximation, the QCF produces an absorption spectrum in very good agreement with the spectrum computed with the exact quantum correction functions, suggesting that classical correlation functions and QCFs can be combined to compute an accurate representation of the third order $\mathrm{cu}-$ mulant contribution. In going from the second to the third order approximations in the cumulant expansion, the third order cumulant contribution corrects the overestimated height of the vibronic shoulder in comparison to the vibronic 0-0 transition, yielding a lineshape in excellent agreement with the exact spectrum. Thus, for this 2-mode model system with moderately non-Gaussian energy gap fluctuations, the third order cumulant correction captures the influence of both Duschinsky mode mixing and the mismatch between ground- and excited state PES curvatures on the linear absorption spectrum, in line with results obtained in Ref. 55 .

Fig. 1b shows the absorptive 2DES signals constructed for the same 2-mode GBOM for three delay times ( $0 \mathrm{fs}$, $30 \mathrm{fs}$, and $60 \mathrm{fs}$ ). The first row contains the spectra constructed in the second order cumulant approximation, whereas the second row shows the third order cumulant approximation, both based on the exact quantum correlation function. At short delay times, the second order cumulant 2DES signal shows a dominant peak at $\omega_{1}=\omega_{3}=3.0 \mathrm{eV}$, corresponding to the $0-0$ transition of the linear absorption spectrum, as well as a number of off-diagonal cross peaks due to vibronic transitions of the 2 -mode system. Adding the third order cumulant correction leads to sharper vibronic peaks for the 2DES signals at $0 \mathrm{fs}$ and $30 \mathrm{fs}$ delay times. For the longer delay time of $60 \mathrm{fs}$, there are more significant changes in the shape of the signal, with off diagonal vibronic features appearing to be shifted towards higher values of $\omega_{1}$ and $\omega_{3}$.

Due to the specific form of the quantum correlation function of the GBOM, the second order cumulant 2DES signals based on purely classical correlation functions and QCFs (See SI Sec. IIIA) nearly match those based on quantum correlation functions. However, as observed in the linear absorption spectra, the same is not true for the third order cumulant correction. The third row of Fig. 1b shows the 2DES signal in the third order cumulant approximation based on classical correlation functions. These spectra show the sharper vibronic features seen with the third order contribution computed with the exact quantum correlation function, but the cross peak at $\omega_{1}=3.0 \mathrm{eV}, \omega_{3}=3.1 \mathrm{eV}$ for $t_{\text {delay }}=30 \mathrm{fs}$ is not exactly reproduced.

To obtain a more detailed analysis of the effects of the third order cumulant correction on the 2DES spectra, we compute the time evolution of the integrated intensities of two points on the 2DES maps (Fig. $1 \mathrm{c}$, marked by boxes in Fig. $1 \mathrm{~b}$ ). The first point is chosen to correspond to the energy of the 0-0 transition for both $\omega_{1}$ and $\omega_{3}$. The second point is a vibronic cross peak with $\omega_{1}=3.15 \mathrm{eV}$, corresponding to a transition between $\nu_{g, 2}^{0}$, the vibrational ground state on the ground state PES for mode 2 and $\nu_{e, 2}^{1}$, the first vibrationally excited state on the excited state PES, and $\omega_{3}=2.84 \mathrm{eV}$, corresponding to a transition between $\nu_{e, 2}^{0}$ and $\nu_{g, 2}^{1}$. Compared to the second order results, the third order cumulant correction computed with the exact quantum correlation function increases the intensity of the 0-0 transition and for the off diagonal cross-peak the amplitude of oscillations is also increased, with a minor shift in the period of oscillation. Compared to the third order cumulant correction computed with the exact quantum correlation function, that computed with the classical correlation function and QCF shows that at $t_{\text {delay }} \approx 50$ fs the amplitude of oscillation in the 0-0 peak signal is underestimated, and the same is true for $t_{\text {delay }} \approx 90 \mathrm{fs}$ and the intensity of the off-diagonal cross peak. However, in agreement with the results presented in Fig. 1 b), the third order cumulant 
a)

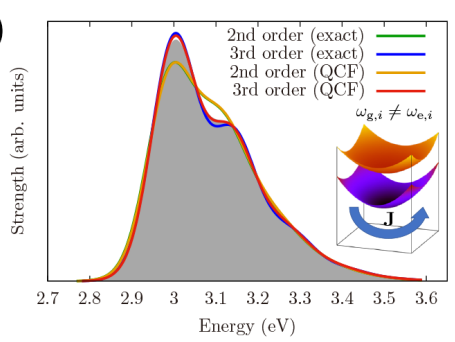

c)

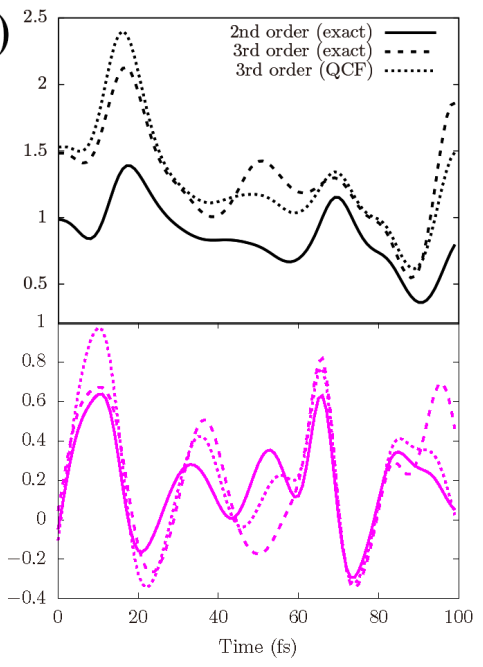

b)

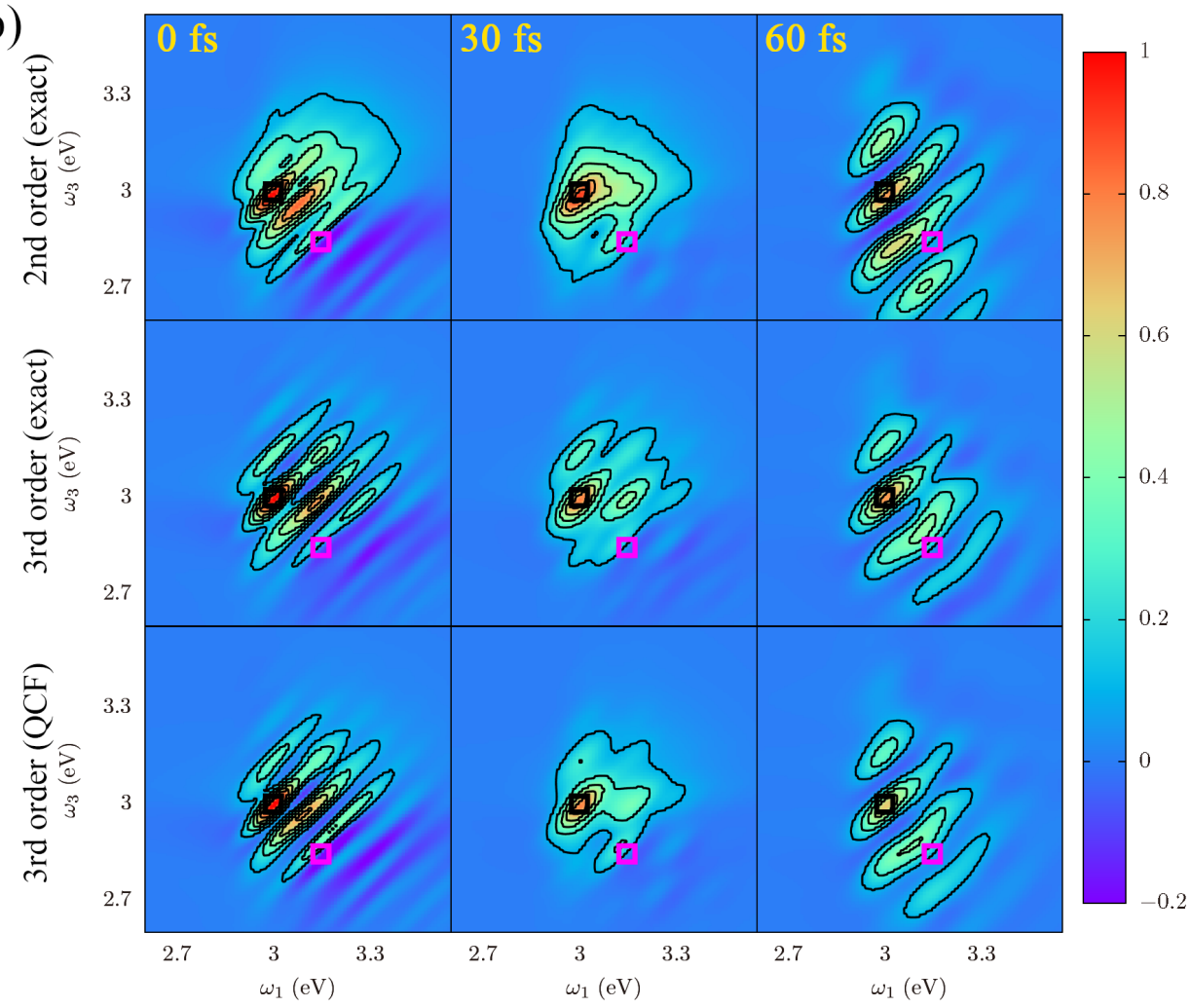

FIG. 1. Nonlinear spectra for a two mode GBOM system with $10^{\circ}$ Duschinksy rotation and an effective solvent broadening of $\lambda_{\text {solv }}=0.0022 \mathrm{Ha}$ : a) Linear spectra in the cumulant approach in comparison with the exact spectrum (shown in gray); b) $2 \mathrm{DES}$ results for $t_{\text {delay }}=0,30$ and $60 \mathrm{fs}$. The first row corresponds to second order cumulant results using the exact quantum correlation function, the second row contains the third order cumulant data for the exact quantum correlation function, and the third row shows the third order cumulant data constructed from the purely classical correlation function using QCFs; c) Time-evolution of integrated intensities for the two boxes shown in the 2DES spectra. Solid lines correspond to exact second order cumulant, dashed lines show exact third order cumulant, and dotted lines correspond to approximate third order cumulant results using QCFs.

contribution constructed using the QCF succeeds in capturing the main corrections of the more exact third order cumulant contribution.

The 2-mode model system of Fig. 1 contains nonGaussian energy gap fluctuations from both the mismatch in ground- and excited state frequencies and the Duschinsky mode mixing. To separate the non-Gaussian effects of mode-mixing from those introduced through a mismatch in ground and excited state vibrational frequencies, we also computed 2DES signals for the same model system with the Duschinksy matrix $\mathbf{J}=\mathbf{I}$, where $\mathbf{I}$ is the identity matrix (see SI Sec. IIIB for this additional analysis). For this simplified model system without Duschinsky rotation effects, the Hamiltonian reduces to the same form as the one considered in Ref. 56. Our analysis shows that in the second order cumulant approximation, both the model system containing a $10^{\circ}$ Duschinksy rotation and the system with $\mathbf{J}=\mathbf{I}$ yield similar results. However, in the third order cumulant approximation the spectra are markedly different. The increased sharpness of the vibronic peaks in the third order cumulant approx- imation, which is also visible in the increased amplitude of oscillatory signals in the time-evolution of integrated intensities, is mainly associated with the mismatch between ground and excited state normal mode frequencies. The overall change in shape of the 2DES signal observed at a longer delay time $t_{\text {delay }}=60$ in Fig. 1 b), where offdiagonal vibronic peaks shift to larger values of $\omega_{1}$ and $\omega_{3}$ in the third order cumulant approximation, is mainly due to the Duschinsky mode-mixing.

\section{B. Nonlinear coupling in a multi-mode system: Nile red}

We next consider the Nile red molecule in benzene. Nile red contains 42 atoms, such that the effective GBOM constructed for this system has 120 modes, all of which are coupled by the Duschinsky rotation matrix (see SI Sec. IIA and B). The effects of the condensed phase environment in the linear and nonlinear spectra are modeled through the spectral density of the Debye form introduced in Eqn. 15, with an effective reorganization en- 
a)

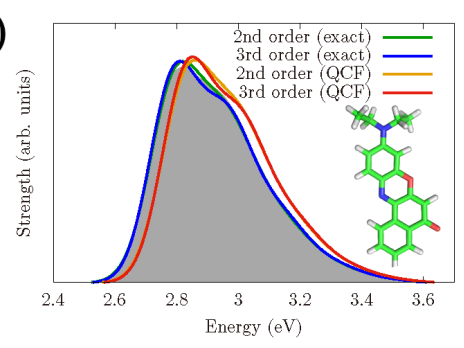

c)

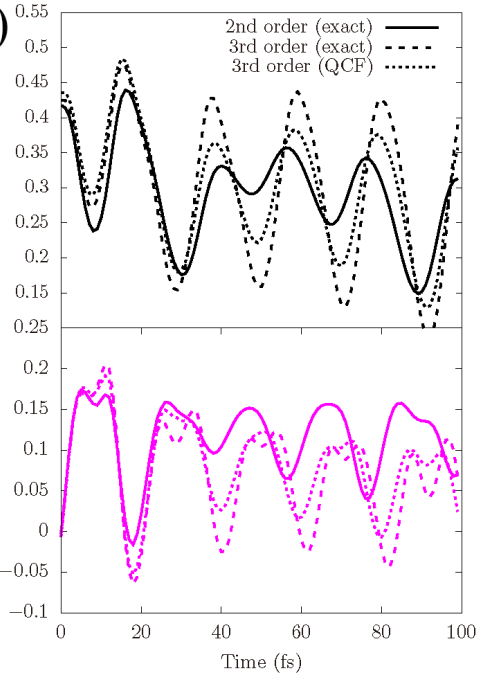

b)

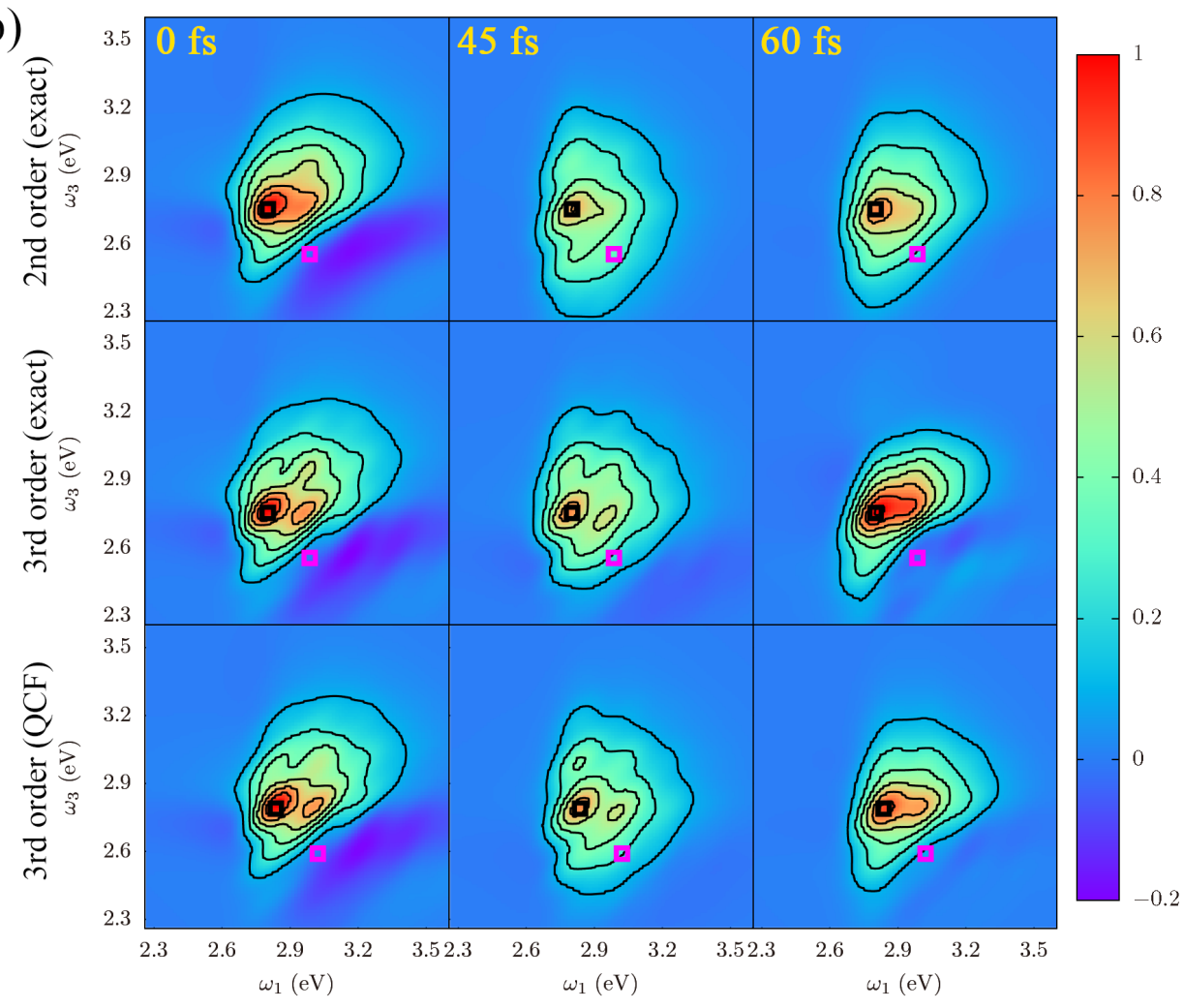

FIG. 2. Results for a GBOM parameterized for Nile red in benzene with an effective solvent broadening of $\lambda_{\text {solv }}=0.0022$ Ha: a) Linear spectra in the cumulant approach in comparison with the exact spectrum (shown in gray) b) 2 DES results for $t_{\text {delay }}=0$, 45 and 60 fs. The first row corresponds to second order cumulant data constructed from the exact quantum correlation function, the second row contains the third order cumulant data constructed from the exact quantum correlation function, and the third row shows the third order cumulant data constructed from the purely classical correlation function using QCFs; c) Time-evolution of integrated intensities for the two boxes shown in the 2DES spectra. Solid lines correspond to second order cumulant constructed from the exact quantum correlation function, dashed lines show third order cumulant constructed from the exact quantum correlation function, and dotted lines correspond to third order cumulant constructed from the purely classical correlation function using QCFs

$\operatorname{ergy} \lambda_{\text {solv }}=0.095 \mathrm{eV}$ chosen such that the GBOM linear spectrum matches the width of the experimental absorption spectrum ${ }^{99}$ (see SI Sec. IV). Although representing the solvent environment through Eqn. 15 yields a model system where the solvent degrees of freedom are completely decoupled from the chromophore degrees of freedom, this approximation performs well in systems where direct solute-solvent interactions are weak, which we have recently shown to be the case for Nile red in benzene. ${ }^{46}$

Fig. 2a shows the linear spectra for Nile red in benzene represented by the GBOM, with the exact spectrum shown in gray and the second and third order spectra as colored lines. The third order cumulant spectrum for this system is almost identical to the second order cumulant spectrum, the only minor difference being that including the third order cumulant term yields a slightly more pronounced vibronic shoulder. The second and third order cumulant spectra constructed from classical correlation functions and the QCFs yield lineshapes that closely agree with the lineshapes produced by the exact quan- tum correlation functions apart from a small red-shift of $\approx 36 \mathrm{meV}$, which is due to the discrepancy between the exact average energy gap $\omega_{\mathrm{eg}}^{\mathrm{av}}=\langle U(\hat{\boldsymbol{q}})\rangle$ and its classical limit. All spectral shapes are in good agreement with the exact GBOM spectrum, suggesting that the second order cumulant approach is a good approximation for the linear absorption spectrum in this system.

Fig. 2b reveals that the nonlinear 2DES signal is more sensitive to the truncation of the cumulant expansion than the linear spectrum, with the second and third order cumulant approximations producing noticeable differences in signal shapes, especially for longer delay times of $t_{\text {delay }}=45 \mathrm{fs}$ and $60 \mathrm{fs}$. For $t_{\text {delay }}=45 \mathrm{fs}$. At these longer times, the second order cumulant approximation produces a spectrum that is elongated along the $\omega_{3}$ axis, whereas including the third order cumulant contribution reduces the elongation and adds more weight to the spectral region with $\omega_{1} \approx 3.2 \mathrm{eV}$. As we found for the simpler 2-mode model, the third order cumulant correction based on purely classical correlation functions and the 
QCF does not exactly reproduce the results using the third order cumulant based on the exact quantum correlation functions, but is in significantly better agreement than the second order cumulant signals.

To analyze the 2DES signals computed with the second and third order cumulant approximations in more detail, we again study the time-evolution of the integrated intensities of two specific regions on the 2DES map (see Fig. 2c). Given the large number of coupled vibrational modes in the system, it is no longer meaningful to choose the points based on a specific transition of a single vibrational mode. Instead, the two points of interest are chosen with reference to the linear absorption spectrum, which shows a peak corresponding to the $0-0$ transition and a vibronic shoulder. The time evolution of the first point of interest corresponds to $\omega_{1}=\omega_{3}=E_{0-0}$, whereas the second point is given by $\omega_{1}=E_{0-0}+\Delta E_{0-1}$, $\omega_{3}=E_{0-0}-\Delta E_{0-1}$ with $\Delta E_{0-1}$ being the energy difference between the 0-0 transition and the vibronic shoulder in the linear absorption spectrum. The first point can thus be interpreted as measuring the time-evolution of the 0-0 transition, whereas the second point probes the time-evolution of the couplings between different vibronic states involved in the vibronic shoulder of the linear absorption spectrum. This simple interpretation of the 2DES signal in terms of a single, effective vibrational mode with frequency equal to $\Delta E_{0-1}$ will likely only be valid in the short time-scale limit of $t_{\text {delay }}$, before coupling to low frequency modes and relaxation through the infinite bath of effective solvent modes becomes significant. Fig. 2c shows that the third order cumulant contribution increases the amplitude of oscillation and leads to a small increase in the period of oscillation in the time evolution of the $E_{0-0}$ signal. This increase in period is more strongly visible in the time evolution of the vibronic cross peak (pink square). Fig. 2c also shows that although the third order correction based on the QCF fails in fully capturing the increase in oscillation amplitude for the $E_{0-0}$ signal, it correctly reproduces the change in oscillation period, both for the $E_{0-0}$ transition and the off-diagonal vibronic cross-peak.

To investigate how much of the third order cumulant contribution to the 2DES signal for Nile red is due to the Duschinsky rotation, we again perform calculations for an effective GBOM with $\mathbf{J}=\mathbf{I}$, i.e. where all modemixing is switched off and the vibrational modes are decoupled. The results in Fig. 3, as computed for the exact quantum correlation function, in comparison with the full GBOM including Duschinsky mode mixing, make it clear that for the Nile red molecule removing the Duschinsky rotation leads to very good agreement between the second and third order cumulant expansion contributions to the 2DES. This good agreement shows that changes to the 2DES upon going from second to third order are dominated by the effect of the Duschinsky rotation over the effect of the mismatch of ground and excited state vibrational frequencies. This finding is confirmed by the timeevolution of integrated intensities (see Fig. 3c). For both the $E_{0-0}$ transition and the off-diagonal vibronic crosspeak the change in oscillation period is almost exclusively due to the Duschinsky mode mixing. Interestingly, the effect on the linear absorption spectrum of ignoring the Duschinsky rotation is very minor, as shown in Fig. 2a.

The results presented for Nile red in benzene demonstrate that third order cumulant corrections can have a significant effect on computed nonlinear optical spectra in realistic systems, even in cases where the linear absorption spectrum is well-described by the second order cumulant approximation. The results also highlight the importance of including Duschinsky rotation effects in modeling 2DES signals, which has been made possible by using the one- and two-time correlation functions for the GBOM derived in our previous work. ${ }^{55} \mathrm{Al}-$ though the QCF for the two-time correlation function ${ }^{65}$ introduces some errors when computing nonlinear spectra from purely classical input, these errors are relatively small and the QCF captures the most important features of the exact third order cumulant contribution.

\section{Dynamic vs. static approaches to obtaining the correlation function: Methylene blue}

The GBOM is a convenient model for many reasons. First, the electronic structure calculations are fairly straightforward because all that is required are the frequencies and coordinates from a ground and excited state geometry optimization. Second, the model accounts for nonlinear coupling from Duschinsky mode mixing and the mismatch of ground- and excited state frequencies. Third, it allows for the computation of exact quantum correlation functions that are inaccessible for more general choices of nuclear Hamiltonian. However, this relatively simple model has two main shortcomings: The first is the restriction to harmonic PESs, meaning that effects due to anharmonicities ${ }^{57,100}$ are ignored. The second is the limitation of the condensed phase environment to a phenomenological spectral density of the Debye form (see Eqn. 15). Because the solvent model requires a phenomenological solvent relaxation time-scale and solvent reorganization energy, ultrafast solvent relaxation processes such as those observed recently in experimental 2DES of BODIPY dyes ${ }^{9}$ cannot be modeled from first principles without experimental input. Furthermore, the solvent model assumes that the condensed phase environment can be fully decoupled from the chromophore degrees of freedom, which is generally not true in systems with strong solute-solvent coupling. An alternate approach for modeling condensed phase systems, albeit computationally considerably more expensive, is to compute classical correlation functions directly from MD trajectories of the chromophore in its complex environment, thus treating chromophore and environment degrees of freedom on the same footing. Here, we showcase the differences between 2DES signals computed from an effective GBOM and from sampling the PES with MD for 
a)

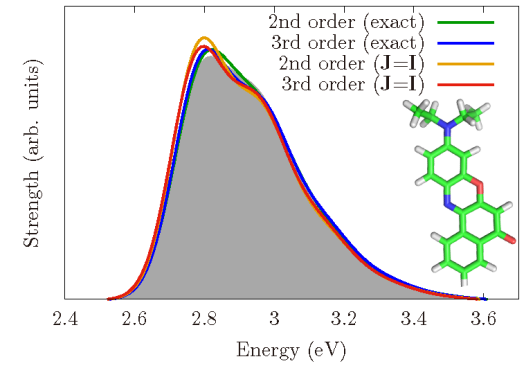

c)

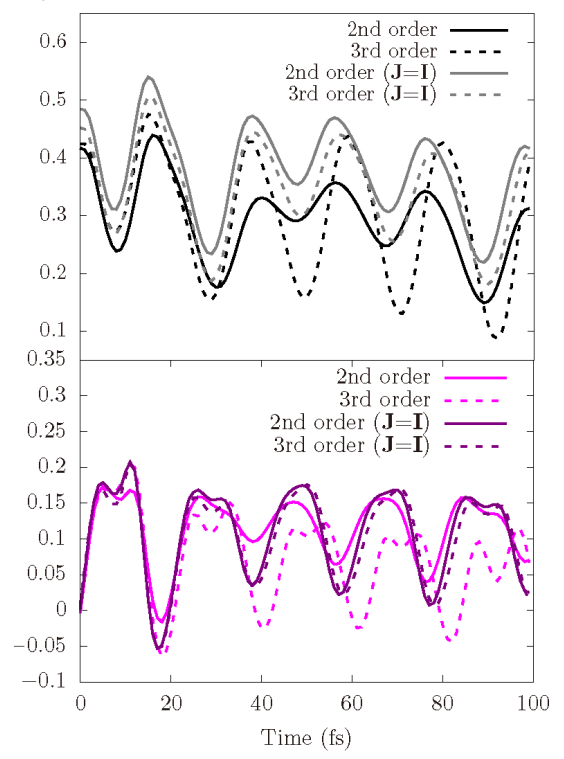

b)

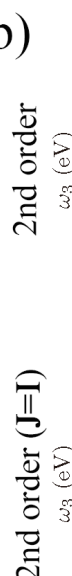

\section{5}

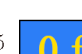

0 fs

$45 \mathrm{fs}$

$60 \mathrm{fs}$

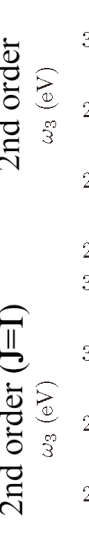

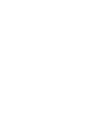
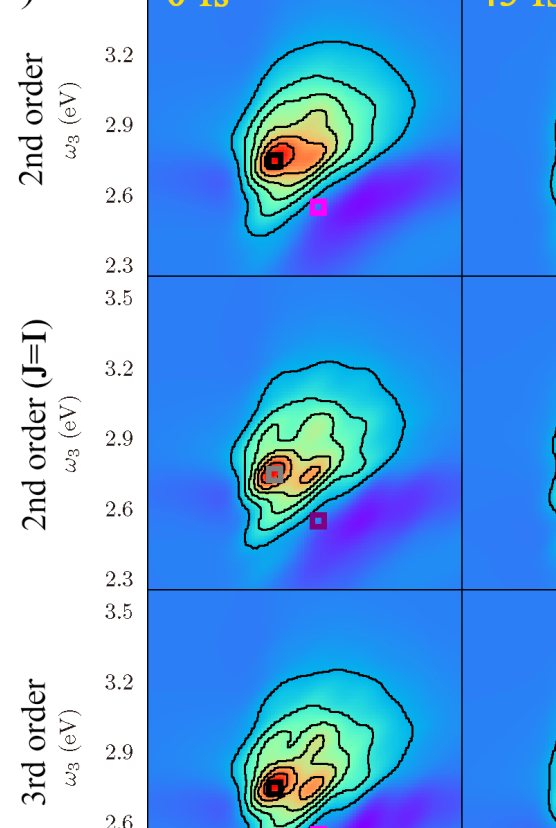

(2) (2)
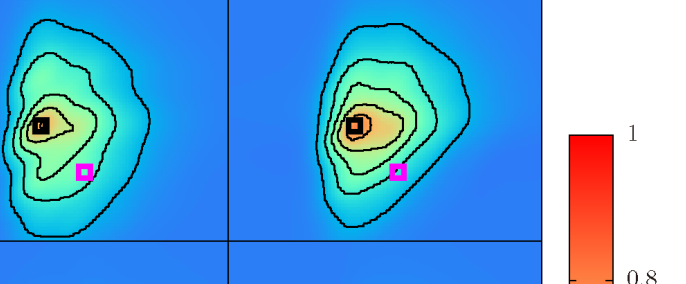

0.8
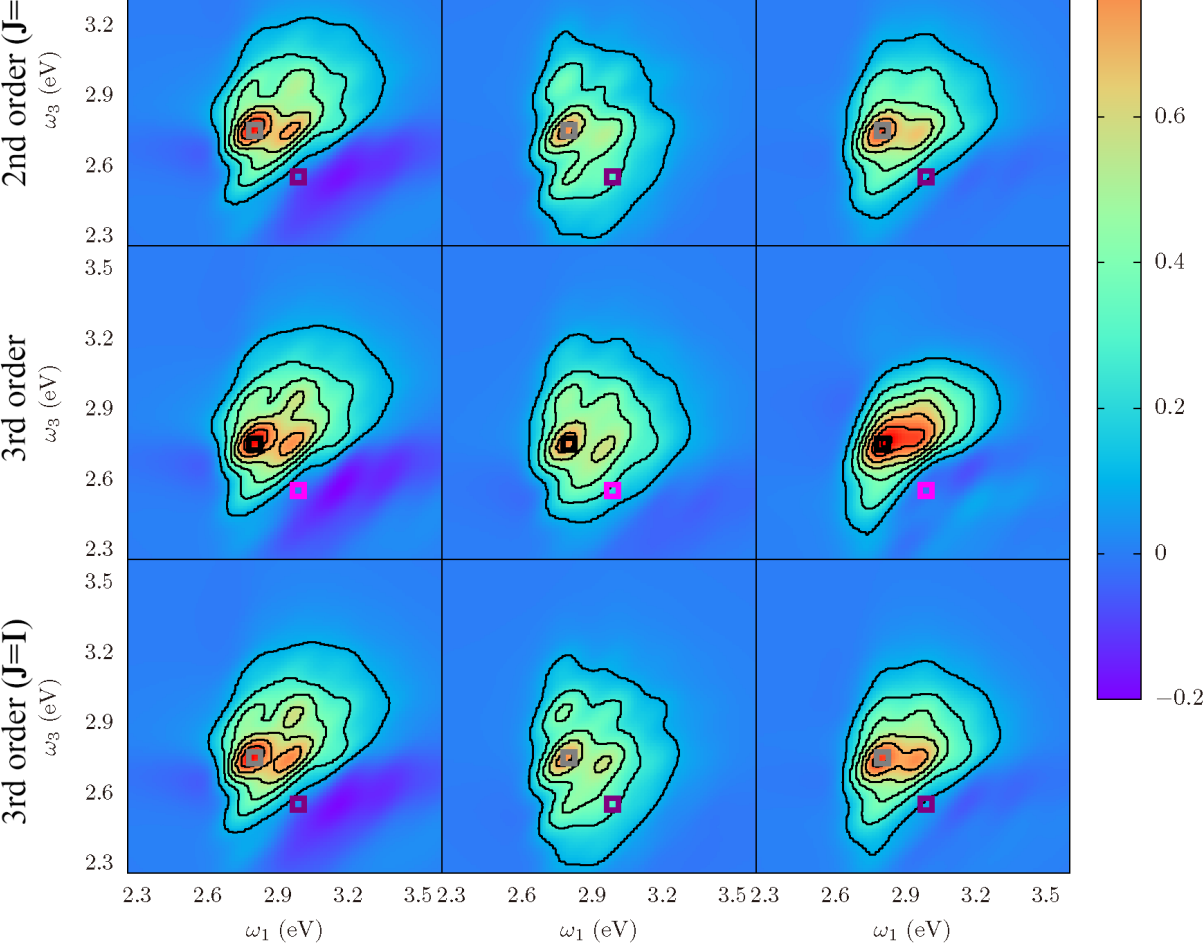

FIG. 3. Results for two GBOMs parameterized for Nile red in benzene, one including Duschinsky mode mixing and one where the Duschinsky matrix is set to the identity matrix a) Linear spectra in the cumulant approach and comparison with the exact spectrum for the full GBOM including Duschinsky rotation (shown in gray) b) $2 \mathrm{DES}$ results for $t_{\mathrm{delay}}=0,45$ and 60 fs. The first and second rows show the second order cumulant results using the exact quantum correlation function, the third and fourth rows show the third order cumulant results using the exact quantum correlation function; c) Time-evolution of integrated intensities for the two boxes shown in the 2DES spectra. Solid lines correspond to exact second order cumulant, dashed lines show exact third order cumulant.

the Methylene blue molecule in water, a system that has been the focus of a number of experimental nonlinear spectroscopy studies. ${ }^{6,7}$

For the GBOM-based 2DES signals, we fix the effective solvent reorganization energy at $\lambda_{\text {solv }}=0.011 \mathrm{eV}$, a value chosen such that the second order cumulant linear absorption spectrum for the GBOM agrees in width with the MD-based second order cumulant spectrum. Furthermore, because the exact QCFs are inaccessible for the MD-based simulations, we compare the MD results to the GBOM spectra based on the classical correlation functions with QCFs. Given the good agreement obtained between the $2 \mathrm{DES}$ signals computed for the exact and the approximate quantum correlation functions for the Nile red molecule represented through a GBOM in Sec. VB, the errors introduced through the QCF are expected to be relatively minor.

The linear absorption spectra in Fig. 4 a) show that the GBOM and the MD sampling of the solvated system yield spectral lineshapes that are in relatively good agreement with each other, both consisting of a dominant 0-0 peak and a vibronic shoulder with relatively low intensity. Similar to the results reported for Nile red in the previous section, the third order cumulant correction has a minor influence on the linear absorption spectrum, yielding a slightly diminished intensity for the 0-0 transition in the GBOM and a small change in the vibronic shoulder for the MD-based spectrum. The MD-based 
a)

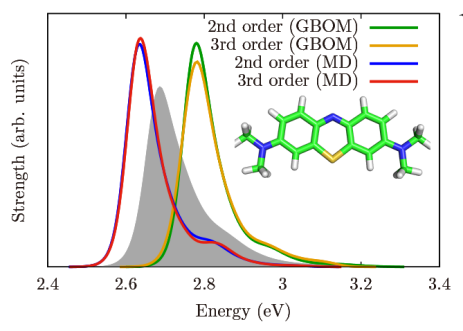

c)

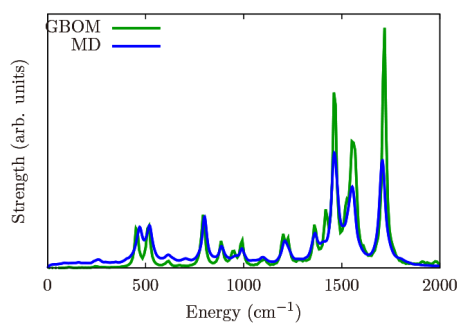

d)

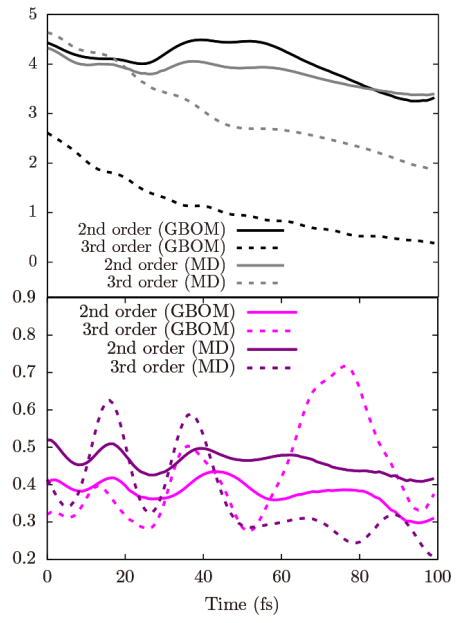

b)

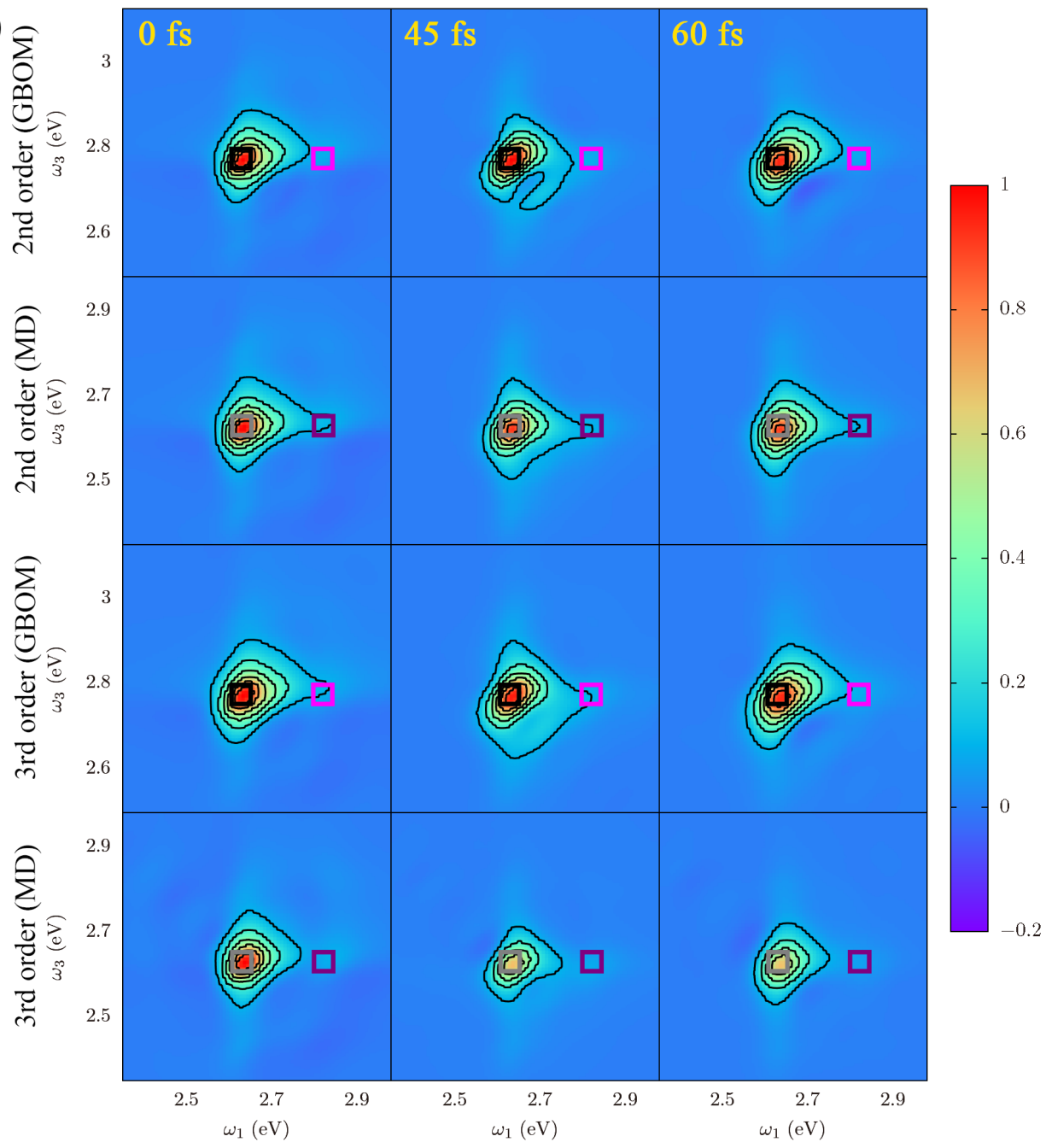

FIG. 4. Results for Methylene blue, both as constructed from an effective GBOM with solvent broadening of $\lambda_{\text {solv }}=0.0004$ Ha and from directly computing the correlation functions from MD: a) Linear spectra in the cumulant approach constructed from classical correlation functions for both GBOM and MD, in comparison to the exact GBOM spectrum (shown in gray); b) 2DES results constructed from classical correlation functions for GBOM and MD, with $t_{\text {delay }}=0$, 45 and 60 fs. The GBOMbased 2DES signals have been shifted in energy by $0.146 \mathrm{eV}$ to allow for an easier comparison with the MD-based spectra; c) Spectral density as constructed from the classical correlation function and QCF for both the GBOM and the MD simulation; d) Time-evolution of integrated intensities for the two boxes shown in the 2DES spectra. Solid lines correspond to second order cumulant, dashed lines show third order cumulant.

spectra are redshifted with respect to the GBOM spectra, which we ascribe to polarization effects of the QM solvent environment. ${ }^{46}$ The GBOM cumulant spectra are blue-shifted in comparison to the exact GBOM spectrum shown in gray, which we again ascribe to the discrepancy between the quantum mechanical thermal average of the energy gap and its classical limit. Since the discrepancy between the two thermal averages can serve as an approximate measure for the degree of non-Gaussian energy gap fluctuations in the GBOM (See SI Sec. IV), and since the observed shift in Fig. 4 a) is considerably larger than for Nile red, we expect that higher order cumulants might play a more significant role in Methylene blue.
For the 2DES (see Fig. 4b), signals computed within the second order cumulant approach show that the GBOM method yields faint off-diagonal cross peaks (see the $t_{\text {delay }}=45 \mathrm{fs}$ signal), which are largely absent in the MD-based second order cumulant spectrum. However, the MD-based spectrum predicts more spectral weight for $\omega_{1} \approx E_{0-0}+\Delta E_{0-1}$ and $\omega_{3} \approx E_{0-0}$ where $\Delta E_{0-1}$ is again taken to denote the energy difference between the $0-0$ peak and the vibronic shoulder in the linear absorption spectrum. When adding the third order cumulant term, the GBOM-based spectrum becomes more delocalized, with increased spectral weight in the $\omega_{1} \approx E_{0-0}+\Delta E_{0-1}, \omega_{3} \approx E_{0-0}$ region, similar to the 
MD-based spectrum in the second order cumulant approximation. For the MD-based third order cumulant spectrum, the most obvious change in the spectral shape is due to loss of intensity in 0-0 transition peak for longer delay times. Overall, we find that although the linear absorption spectra produced by the GBOM Hamiltonian and the MD sampling of the system in its complex environment are very similar, the nonlinear spectra reveal subtle differences in the coupling of the electronic state to high-frequency vibrational modes and low frequency solvent relaxation.

This interpretation is confirmed by comparing the spectral densities obtained from the GBOM and the MD data (see Fig. $4 \mathrm{c}$ )). Although the peak positions of most mid- to high frequency vibronic peaks agree, suggesting that the ground- and excited state PESs for the most dominant vibrational modes are indeed close to harmonic, the intensity of vibronic peaks differs, especially in the $\omega>1400 \mathrm{~cm}^{-1}$ region of the spectral density (See also SI Sec. VC). For the three most dominant vibronic peaks, the intensities for the MD model are lower than for the pure GBOM. This rescaling of intensities is a direct manifestation of solvent polarization effects influencing high frequency vibronic couplings; we observed a similar effect for model chromophores of the green fluorescent protein (GFP) and photoactive yellow protein (PYP) chromophores in water. ${ }^{46}$ In contrast, the low frequency region of the spectral density is strongly underestimated in the GBOM approach, which can be ascribed to the lack of collective chromophore-environment degrees of freedom coupling to the energy gap.

To analyze the differences between the 2DES signals predicted by the GBOM and the MD in more detail, we again compare the time evolution of integrated intensities, this time for the 0-0 transition and the $\omega_{1}=$ $E_{0-0}+\Delta E_{0-1}, \omega_{3}=E_{0-0}$ points on the 2DES map (see Fig. 4d). The differences in time evolution in the second order cumulant approximation between the GBOM and the MD model are relatively small and mainly consist of a smaller spectral weight on the $\omega_{1}=E_{0-0}+\Delta E_{0-1}$, $\omega_{3}=E_{0-0}$ transition for the GBOM. The differences in the third order cumulant approach are considerably more pronounced. Both the GBOM and the MD model predict a much more rapid decay of the 0-0 signal for increasing delay times than was obtained in the second order cumulant approach. For the off-diagonal vibronic peak, the amplitude of oscillation increases strongly in going from second to the third order cumulant approximation. However, for the MD model, this increase in amplitude is confined to short delay times, whereas for the GBOM the amplitude increases with increasing delay time. Thus, although the second order cumulant spectra show similar trends owing to the relatively close agreement of the spectral densities for the two models, the third order cumulant corrections are very different, suggesting that the nonlinear couplings described by the two models differ significantly.

A more detailed picture of how the MD sampling of the chromophore within its complex environment differs from the GBOM description is obtained by analyzing which parts of the third order cumulant correction in the MD model are caused by direct solvent interactions (see analysis in SI Sec. VC). We refer to solvent interactions as "direct" if they are due to a direct coupling of the electronic excited state of the chromophore to surrounding solvent molecules. Conversely, we refer to solventinduced changes in the motion of the chromophore as "indirect" solvent interactions. We are able to differentiate between direct and indirect solvent interaction effects in the cumulant approach by taking a single MD trajectory and calculating correlation functions for vertical excitation energies computed for both the fully solvated system and the same snapshots where the solvent environment is stripped away. For Methylene blue, we find that most of the third order cumulant correction to 2DES signals originates from direct solute-solvent interactions, i.e. coupling of electronic polarization of the environment to the electronic excited state of the chromophore. In our recent study ${ }^{46}$ we showed that representing the condensed phase environment explicitly at the QM level in an MD-based cumulant approach can be crucial for obtaining reliable spectral densities and linear absorption spectra. Here, we show that nonlinear optical signals are also highly sensitive to the treatment of the condensed phase environment, especially when including contributions from higher order cumulants.

\section{CONCLUSIONS}

Calculating accurate nonlinear optical spectra from first principles is important for connecting spectroscopic experiments to the electronic dynamics of chromophores in complex environments, ranging from ultrafast solventdriven relaxation effects in solvated dyes to energy transfer in pigment-protein complexes. Here, we investigated the performance of the cumulant-based approach in calculating nonlinear spectra, with a special focus on the third order cumulant term that approximately captures effects introduced through a mismatch between curvatures of the ground- and excited state potential energy surfaces as well as Duschinsky mode mixing. We derived a general expression for the third order cumulant correction to the nonlinear response function and applied it to a GBOM system with harmonic potential energy surfaces. In this simplified model that can be solved exactly for linear absorption, we show that adding the third order contribution improves the accuracy of the linear absorption spectrum and that it has a significant effect on the 2DES signal, likely leading to improved accuracy for short timescales. We find that the third order contribution substantially changes simulated nonlinear spectral features even in systems where the linear absorption spectrum is well described by a second order cumulant expansion. We have also shown that for this simplified harmonic model, the nonlinear spectra computed 
in the second- and third-order cumulant approximation can be well reproduced from purely classical dynamics using quantum correction factors, extending a similar observation made previously in the context of linear spectroscopy.

One of the features of our newly derived expressions for the third order cumulant correction to the 2DES signal is the ability to account for Duschinsky mode mixing within the GBOM. We have found for both a simple 2 mode system and for the Nile Red chromophore that 2DES results computed within the second order cumulant approximation show very little difference upon adding Duschinsky mode mixing, but that this is no longer the case within the third order cumulant approximation. The 2DES computed within the third order cumulant approximation showed that the Duschinsky mode mixing led to off-diagonal vibronic peaks shifting to higher energies for the 2 mode system and led to an increase in the amplitude of oscillation and an increase in the period of oscillation in the time evolution of the vibronic coherences for Nile Red.

The harmonic model considered in this work can be straightforwardly constructed using standard quantum chemistry software packages by computing vibrational normal modes on the ground and excited state potential energy surface, enabling a computationally efficient route to interpreting nonlinear optical spectra of solvated dyes. We expect this approach to perform well for rigid chromophores that couple weakly to their condensed phase environment, especially in the limit of short delay times where the electronic excited state relaxes in the Condon region. For systems with low frequency anharmonic motion or chromophores embedded in strongly interacting condensed phase environments, the simplified harmonic model is likely to perform poorly. In these systems, a cumulant approach based on directly sampling the (potentially anharmonic) potential energy surface of the system embedded in its atomistic condensed phase environment using molecular dynamics will likely yield improved results. Although this approach is computationally more expensive than constructing a simple model system based on ground- and excited state vibrational normal modes, it has the advantage of approximately capturing both the anharmonic nature of the potential energy surface and the influence of specific environmental interactions on excited state relaxation. With the development of highly efficient electronic structure methods and increasing availability of high-performance computing resources, direct applications of the cumulant approach outlined in this work to the nonlinear optical response of complex condensed phase systems are now possible.

\section{ACKNOWLEDGMENTS}

The data that support the findings of this study are available from the corresponding authors upon request. This work was supported by the Department of En- ergy Basic Energy Sciences CTC and CPIMS programs (Grant No DE-SC0019053). Calculations were performed using the MERCED computational resource, supported by the National Science Foundation Major Research Instrumentation program (ACI-1429783).

${ }^{1}$ G. D. Scholes, G. R. Fleming, A. Olaya-Castro, and R. van Grondelle, "Lessons from nature about solar light harvesting," Nat. Chem. 3, 763-774 (2011).

${ }^{2}$ G. S. Schlau-Cohen, "Principles of light harvesting from single photosynthetic complexes," Interface Focus 5, 20140088 (2015).

${ }^{3}$ H. L. Luk, F. Melaccio, S. Rinaldi, S. Gozem, and M. Olivucci, "Molecular bases for the selection of the chromophore of animal rhodopsins," Proc. Natl. Acad. Sci. U.S.A. 112, 15297-15302 (2015).

${ }^{4}$ M. K. Brennaman, R. J. Dillon, L. Alibabaei, M. K. Gish, C. J. Dares, D. L. Ashford, R. L. House, G. J. Meyer, J. M. Papanikolas, and T. J. Meyer, "Finding the way to solar fuels with dye-sensitized photoelectrosynthesis cells," J. Am. Chem. Soc. 138, 13085-13102 (2016).

${ }^{5}$ S. Gulati, B. Jastrzebska, S. Banerjee, Á. L. Placeres, P. Miszta, S. Gao, K. Gunderson, G. P. Tochtrop, S. Filipek, K. Katayama, P. D. Kiser, M. Mogi, P. L. Stewart, and K. Palczewski, "Photocyclic behavior of rhodopsin induced by an atypical isomerization mechanism," Proc. Natl. Acad. Sci. U.S.A. 114, E2608E2615 (2017).

${ }^{6}$ J. C. Dean, S. Rafiq, D. G. Oblinsky, E. Cassette, C. C. Jumper, and G. D. Scholes, "Broadband Transient Absorption and TwoDimensional Electronic Spectroscopy of Methylene Blue," J. Phys. Chem. A 119, 9098-9108 (2015).

${ }^{7}$ J. C. Dean, D. G. Oblinsky, S. Rafiq, and G. D. Scholes, "Methylene blue exciton states steer nonradiative relaxation: Ultrafast spectroscopy of methylene blue dimer," J. Phys. Chem. B 120, 440-454 (2016).

${ }^{8}$ J. C. Dean and G. D. Scholes, "Coherence spectroscopy in the condensed phase: Insights into molecular structure, environment, and interactions," Acc. Chem. Res. 50, 2746-2755 (2017).

${ }^{9}$ Y. Lee, S. Das, R. M. Malamakal, S. Meloni, D. M. Chenoweth, and J. M. Anna, "Ultrafast solvation dynamics and vibrational coherences of halogenated boron-dipyrromethene derivatives revealed through two-dimensional electronic spectroscopy," J. Am. Chem. Soc. 139, 14733-14742 (2017).

${ }^{10}$ L. Bolzonello, A. Polo, A. Volpato, E. Meneghin, M. Cordaro, M. Trapani, M. Fortino, A. Pedone, M. A. Castriciano, and E. Collini, "Two-dimensional electronic spectroscopy reveals dynamics and mechanisms of solvent-driven inertial relaxation in polar bodipy dyes," J. Phys. Chem. Lett. 9, 1079-1085 (2018).

${ }^{11}$ G. S. Engel, T. R. Calhoun, E. L. Read, T.-K. Ahn, T. Mančal, Y.-C. Cheng, R. E. Blankenship, and G. R. Fleming, "Evidence for wavelike energy transfer through quantum coherence in photosynthetic systems," Nature 446, 782-786 (2007).

${ }^{12}$ T. R. Calhoun, N. S. Ginsberg, G. S. Schlau-Cohen, Y.-C. Cheng, M. Ballottari, R. Bassi, and G. R. Fleming, "Quantum coherence enabled determination of the energy landscape in light-harvesting complex ii," J. Phys. Chem. B 113, 1629116295 (2009).

${ }^{13}$ J. Dostál, J. Pšenčík, and J. Zigmantas, "In situ mapping of the energy flow through the entire photosynthetic apparatus," Nat. Chem. 8, 705-710 (2016).

${ }^{14}$ H.-G. Duan, V. I. Prokhorenko, E. Wientjes, R. Croce, M. Thorwart, and R. J. D. Miller, "Primary charge separation in the photosystem ii reaction center revealed by a global analysis of the two-dimensional electronic spectra," Sci. Rep. 7, 12347 (2017).

${ }^{15}$ M. Maiuri, E. E. Ostroumov, R. G. Saer, R. E. Blankenship, and G. D. Scholes, "Coherent wavepackets in the fenna-matthews-olson complex are robust to excitonicstructure perturbations caused by mutagenesis," Nat. Chem. 10, 177-183 (2018). 
${ }^{16}$ T. J. Martínez and R. D. Levine, "First-principles molecular dynamics on multiple electronic states: A case study of nai," J. Chem. Phys. 105, 6334-6341 (1996).

${ }^{17}$ T. Vreven, F. Bernardi, M. Garavelli, M. Olivucci, M. A. Robb, and H. B. Schlegel, "Ab initio photoisomerization dynamics of a simple retinal chromophore model," J. Am. Chem. Soc. 119, 12687-12688 (1997).

${ }^{18}$ M. H. Beck, A. Jäckle, G. A. Worth, and H. D. Meyer, "The multiconfiguration time-dependent hartree (mctdh) method: A highly efficient algorithm for propagating wavepackets," Phys. Rep. 324, 1-105 (2000)

${ }^{19}$ B. F. E. Curchod and T. J. Martínez, "Ab initio nonadiabatic quantum molecular dynamics," Chem. Rev. 118, 3305-3336 (2018).

${ }^{20}$ R. Cammi, S. Corni, B. Mennucci, and J. Tomasi, "Electronic excitation energies of molecules in solution: State specific and linear response methods for nonequilibrium continuum solvation models," J. Chem. Phys. 122, 104513 (2005).

${ }^{21}$ B. Mennucci, "Polarizable continuum model," Wiley Interdisciplinary Reviews: Computational Molecular Science 2, 386-404 (2012).

${ }^{22}$ F. Lipparini and B. Mennucci, "Perspective: Polarizable continuum models for quantum-mechanical descriptions," J. Chem. Phys. 144, 160901 (2016).

${ }^{23}$ C. Olbrich, J. Strümpfer, K. Schulten, and U. Kleinekathöfer, "Theory and Simulation of the Environmental Effects on FMO Electronic Transitions," J. Phys. Chem. Lett. 14, 1771-1776 (2011).

${ }^{24}$ S. Shim, P. Rebentrost, S. Valleau, and A. Aspuru-Guzik, "Atomistic Study of the Long-Lived Quantum Coherences in the Fenna-Matthews-Olson Complex," Biophys. J. 102, 649660 (2012).

${ }^{25}$ M. K. Lee, K. B. Bravaya, and D. F. Coker, "First-Principles Models for Biological Light-Harvesting: Phycobiliprotein Complexes from Cryptophyte Algae," J. Am. Chem. Soc. 139, 78031814 (2017).

${ }^{26}$ M. I. Mallus, Y. Shakya, J. D. Prajapati, and U. Kleinekathöfer, "Environmental effects on the dynamics in the light-harvesting complexes LH2 and LH3 based on molecular simulations," Chem. Phys. 515, 141-151 (2018).

${ }^{27}$ S. M. Blau, I. G. Bennett, C. Kreisbeck, G. D. Scholes, and A. Aspuru-Guzik, "Local protein solvation drives direct downconversion in phycobiliprotein PC645 via incoherent vibronic transport," Proc. Natl. Acad. Sci. U.S.A. 115, E3342-E3350 (2018).

${ }^{28}$ C. M. Isborn, A. W. Götz, M. A. Clark, R. C. Walker, and T. J. Martínez, "Electronic absorption spectra from $\mathrm{mm}$ and ab initio $\mathrm{qm} / \mathrm{mm}$ molecular dynamics: Environmental effects on the absorption spectrum of photoactive yellow protein," J. Chem. Theory Comput. 8, 5092-5106 (2012).

${ }^{29}$ R. Crespo-Otero and M. Barbatti, "Spectrum simulation and decomposition with nuclear ensemble: formal derivation and application to benzene, furan and 2-phenylfuran," Theor. Chem. Acc. 131, 1237 (2012).

${ }^{30}$ F. J. A. Ferrer, M. D. Davari, D. Morozov, G. Groenhof, and F. Santoro, "The lineshape of the electronic spectrum of the green fluorescent protein chromophore, part ii: Solution phase," ChemPhysChem 15, 3246-3257 (2014).

${ }^{31}$ X. Ge, I. Timrov, S. Binnie, A. Biancardi, A. Calzolari, and S. Baroni, "Accurate and inexpensive prediction of the color optical properties of anthocyanins in solution," J. Phys. Chem. A 119, 3816-3822 (2015).

${ }^{32}$ A. V. Marenich, C. J. Cramer, and D. G. Truhlar, "Electronic absorption spectra and solvatochromic shifts by the vertical excitation model: Solvated clusters and molecular dynamics sampling," J. Phys. Chem. B 119, 958-967 (2015).

${ }^{33}$ J. Cerezo, F. J. Avila Ferrer, G. Prampolini, and F. Santoro, "Modeling solvent broadening on the vibronic spectra of a series of coumarin dyes. from implicit to explicit solvent models," J. Chem. Theory Comput. 11, 5810-5825 (2015).
${ }^{34}$ J. M. Milanese, M. R. Provorse, E. Alameda, and C. M. Isborn, "Convergence of computed aqueous absorption spectra with explicit quantum mechanical solvent," J. Chem. Theory Comput. 13, 2159-2171 (2017).

${ }^{35}$ T. J. Zuehlsdorff, P. D. Haynes, M. C. Payne, and N. D. M. Hine, "Predicting solvatochromic shifts and colours of a solvated organic dye: The example of nile red," J. Chem. Phys. 146, 124504 (2017).

${ }^{36}$ T. J. Zuehlsdorff and C. M. Isborn, "Modeling absorption spectra of molecules in solution," Int. J. Quantum Chem. 119, e25719 (2019)

${ }^{37}$ S. V. Shedge, T. J. Zuehlsdorff, M. J. Servis, A. E. Clark, and C. M. Isborn, "Effect of ions on the optical absorption spectra of aqueously solvated chromophores," J. Phys. Chem. A 123, 6175-6184 (2019).

${ }^{38} \mathrm{M}$. Retegan, F. Neese, and D. A. Pantazis, "Convergence of $\mathrm{qm} / \mathrm{mm}$ and cluster models for the spectroscopic properties of the oxygen-evolving complex in photosystem ii," J. Chem. Theory Comput. 9, 3832-3842 (2013).

${ }^{39}$ O. Valsson, P. Campomanes, I. Tavernelli, U. Rothlisberger, and C. Filippi, "Rhodopsin absorption from first principles: Bypassing common pitfalls," J. Chem. Theory Comput. 9, 24412454 (2013).

${ }^{40}$ D. J. Cole, A. W. Chin, N. D. M. Hine, P. D. Haynes, and M. C. Payne, "Toward ab initio optical spectroscopy of the fennamatthews-olson complex," J. Phys. Chem. Lett. 4, 4206-4212 (2013).

${ }^{41}$ M. R. Provorse, T. Peev, C. Xiong, and C. M. Isborn, "Convergence of excitation energies in mixed quantum and classical solvent: Comparison of continuum and point charge models," J. Phys. Chem. B 120, 12148-12159 (2016), pMID: 27797196.

${ }^{42}$ T. J. Zuehlsdorff, P. D. Haynes, F. Hanke, M. C. Payne, and N. D. M. Hine, "Solvent effects on electronic excitations of an organic chromophore," J. Chem. Theory Comput. 12, 18531861 (2016).

${ }^{43}$ C. M. Isborn, N. Luehr, I. S. Ufimtsev, and T. J. Martínez, "Excited-State Electronic Structure with Configuration Interaction Singles and Tamm-Dancoff Time-Dependent Density Fucntional Theory on Graphical Processing Units," J. Chem. Theory Comput. 7, 1814-1823 (2011).

${ }^{44}$ T. J. Zuehlsdorff, N. D. M. Hine, J. S. Spencer, N. M. Harrison, D. J. Riley, and P. D. Haynes, "Linear-scaling time-dependent density-functional theory in the linear response formalism," J. Chem. Phys 139, 064104 (2013).

${ }^{45}$ T. J. Zuehlsdorff, N. D. M. Hine, M. C. Payne, and P. D. Haynes, "Linear-scaling time-dependent density-functional theory beyond the Tamm-Dancoff approximation: Obtaining efficiency and accuracy with in situ optimised local orbitals," J. Chem. Phys 143, 204107 (2015).

${ }^{46}$ T. J. Zuehlsdorff, H. Hong, L. Shi, and C. M. Isborn, "Influence of Electronic Polarization on the Spectral Density," J. Phys. Chem. B 124, 531-543 (2020).

${ }^{47}$ S. Mukamel, Principles of Nonlinear Optical Spectroscopy (Oxford University Press, New York, 1995).

${ }^{48}$ M. C. Zwier, J. M. Shorb, and B. P. Krueger, "Hybrid Molecular Dynamics-Quantum Mechanics Simulations of Solute Spectral Properties in the Condensed Phase: Evaluation of Simulation Parameters," J. Comput. Chem. 28, 1572-1581 (2007).

${ }^{49}$ S. Valleau, A. Eisfeld, and A. Aspuru-Guzik, "On the alternatives for bath correlators and spectral densities from mixed quantum-classical simulations," J. Chem. Phys. 137, 224103 (2012).

${ }^{50}$ D. Loco, S. Jurinovich, L. Cupellini, M. F. S. J. Menger, and B. Mennucci, "The modeling of the absorption lineshape for embedded molecules through a polarizable QM/MM approach," Photochem. Photobiol. Sci. 17, 552-560 (2018).

${ }^{51}$ J. S. Bader and B. J. Berne, "Quantum and classical relaxation rates from classical simulations," J. Chem. Phys. 100, 8359 (1994). 
${ }^{52}$ S. A. Egorov, K. F. Everitt, and J. L. Skinner, "Quantum Dynamics and Vibrational Relaxation," J. Phys. Chem. A 103, 9494-9499 (1999).

${ }^{53}$ H. Kim and P. J. Rossky, "Evaluation of Quantum Correlation Functions from Classical Data," J. Phys. Chem. B 106, 8240 (2002).

${ }^{54}$ A. Anda, L. De Vico, T. Hansen, and Abramavičius, "Absorption and fluorescence lineshape theory for polynomial potentials," J. Chem. Theory Comput. 12, 5979-5989 (2016).

${ }^{55}$ T. J. Zuehlsdorff, A. Montoya-Castillo, J. A. Napoli, T. E. Markland, and C. M. Isborn, "Optical spectra in the condensed phase: Capturing anharmonic and vibronic features using dynamic and static approaches," J. Chem. Phys 151, 074111 (2019).

${ }^{56}$ A. F. Fidler and G. S. Engel, "Nonlinear spectroscopy theory of displaced harmonic oscillators with differing curvatures: A correlation function approach," J. Phys. Chem. A 117, 94449453 (2013).

${ }^{57}$ A. Anda, D. Abramavičius, and T. Hansen, "Two-dimensional electronic spectroscopy of anharmonic molecular potentials," Phys. Chem. Chem. Phys. 20, 1642-1652 (2018).

${ }^{58}$ C. Kreisbeck and T. Kramer, "Long-Lived Electronic Coherence in Dissipative Exciton Dynamics of Light-Harvesting Complexes," J. Phys. Chem. Lett. 3, 2828-2833 (2012).

${ }^{59}$ L. Viani, M. Corbella, C. Curutchet, E. J. O'Reilly, A. OlayaCastro, and B. Mennucci, "Molecular basis of the exciton-phonon interactions in the PE545 light-harvesting complex," Phys. Chem. Chem. Phys. 16, 16302 (2014).

${ }^{60}$ M. K. Lee and D. F. Coker, "Modeling Electronic-Nuclear Interactions for Excitation Energy Transfer Processes in LightHarvesting Complexes," J. Phys. Chem. Lett. 7, 3171-3178 (2016).

${ }^{61}$ M. K. Lee, P. Huo, and D. F. Coker, "Semiclassical Path Integral Dynamics: Photosynthetic Energy Transfer with Realistic Environment Interactions," Annu. Rev. Phys. Chem. 67, 639668 (2016).

${ }^{62} \mathrm{~S}$. J. Jang and B. Mennucci, "Delocalized excitons in natural light-harvesting complexes," Rev. Mod. Phys. 90, 035003 (2018).

${ }^{63}$ O. Rancova and D. Abramavičius, "Static and dynamic disorder in bacterial light-harvesting complex lh2: A 2des simulation study," J. Phys. Chem. B 118, 7533-7540 (2014).

${ }^{64}$ F. Duschinsky, ", Acta Physicochim. URSS. 7, 411 (1937).

${ }^{65}$ K. A. Jung, P. E. Videla, and V. S. Batista, "Inclusion of nuclear quantum effects for simulations of nonlinear spectroscopy," J. Chem. Phys. 148, 244105 (2018).

${ }^{66}$ A. Warshel and M. Karplus, "Vibrational structure of electronic transitions in conjugated molecules," Chem. Phys. Lett. 17, 714 (1972)

${ }^{67}$ L. S. Cederbaum and W. Domcke, "A many-body approach to the vibrational structure in molecular electronic spectra. I. Theory," J. Chem. Phys. 64, 603 (1976).

${ }^{68}$ E. V. Doktorov, I. A. Malkin, and V. I. Manko, "Dynamical symmetry of vibronic transitions in polyatomic molecules and the Franck-Condon principle," J. Mol. Spectrosc. 64, 302-326 (1977).

${ }^{69} \mathrm{P}$. T. Ruhrhoff, "Recursion relations for multi-dimensional Franck-Condon overlap integrals," Chem. Phys. 186, 335-374 (1994).

${ }^{70}$ F. Santoro, A. Lami, R. Improta, J. Bloino, and V. Barone, "Effective method for the computation of optical spectra of large molecules at finite temperature including the duschinsky and herzberg-teller effect: The qx band of porphyrin as a case study," J. Chem. Phys. 128, 224311 (2008).

${ }^{71}$ J. Bloino, M. Biczysko, F. Santoro, and V. Barone, "General approach to compute vibrationally resolved one-photon electronic spectra," J. Chem. Theory Comput. 6, 1256-1274 (2010).

${ }^{72}$ Y. Niu, Q. Peng, C. Deng, X. Gao, and Z. Shuai, "Theory of Excited State Decays and Optical Spectra: Application to Polyatomic Molecules," J. Phys. Chem. A 114, 7817-7831 (2010).
${ }^{73}$ A. Baiardi, J. Bloino, and V. Barone, "General time dependent approach to vibronic spectroscopy including franckcondon, herzberg-teller, and duschinky effects," J. Chem. Theory Comput. 9, 4097-4115 (2013).

${ }^{74}$ F. Santoro and D. Jaquemin, "Going beyond the vertical approximation with time-dependent density functional theory," Wiley Interdiscip. Rev.: Comput. Mol. Sci. 6, 460-486 (2016).

${ }^{75}$ B. de Souza, F. Neese, and R. Izsák, "On the theoretical prediction of fluorescence rates from first principles using the path integral approach," J. Chem. Phys. 148, 034104 (2018).

${ }^{76}$ E. Condon, "A Theory of Intensity Distribution in Band Systems," Phys. Rev. 28, 1182-1201 (1926).

${ }^{77}$ E. Condon, "Nuclear Motion Associated with Electron Transitions in Diatomic molecles," Phys. Rev. 32, 858-872 (1928).

${ }^{78}$ A. Nenov, A. Giussani, B. P. Fingerhut, I. Rivalta, E. Dumont, S. Mukamel, and M. Garavelli, "Spectral lineshapes in nonlinear electronic spectroscopy," Phys. Chem. Chem. Phys. 17, 3092530936 (2015).

${ }^{79}$ M. Kowalewski, B. P. Fingerhut, K. E. Dorfman, K. Bennett, and S. Mukamel, "Simulating coherent multidimensional spectroscopy of nonadiabatic molecular processes: From the infrared to the x-ray regime," Chem. Rev. 117, 12165-12226 (2017).

${ }^{80}$ I. R. Craig and D. E. Manolopoulos, "Quantum statistics and classical mechanics: Real time correlation functions from ring polymer molecular dynamics," J. Chem. Phys. 121, 3368 (2004).

${ }^{81}$ R. Ramirez, T. Lopez-Ciudad, P. K. P, and D. Marx, "Quantum corrections to classical time-correlation functions: Hydrogen bonding and anharmonic floppy modes," J. Chem. Phys. 121, 3973 (2004).

${ }^{82}$ F. J. Avila Ferrer, M. D. Davari, D. Morozov, G. Groenhof, and F. Santoro, "The lineshape of the electronic spectrum of the green fluorescent protein chromophore, part ii: Solution phase," Chem. Phys. Chem. 15, 3246-3257 (2014).

${ }^{83}$ R. Improta, F. J. Avila Ferrer, E. Standardo, and F. Santoro, "Quantum-classical calculation of the absorption and emission spectral shapes of oligothiophenes at low and room temperature by first-principle calculations," Chem. Phys. Chem. 15, 33203333 (2014).

${ }^{84}$ T. J. Zuehlsdorff, J. A. Napoli, J. M. Milanese, T. E. Markland, and C. M. Isborn, "Unraveling electronic absorption spectra using nuclear quantum effects: Photoactive yellow protein and green fluorescent protein chromophores in water," J. Chem. Phys. 149, 024107 (2018).

${ }^{85}$ T. J. Zuehlsdorff and C. M. Isborn, "Combining the ensemble and Franck-Condon approaches for calculating spectral shapes of molecules in solution," J. Chem. Phys. 148, 024110 (2018).

${ }^{86}$ M. Forino, E. Collini, A. Pedone, and J. Bloino, "Role of specific solute-solvent interactions on the photophysical properties of distyryl substituted bodipy derivatives," Phys. Chem. Chem. Phys., Advance Article (2020).

${ }^{87}$ A. Baiardi, J. Bloino, and V. Barone, "General formulation of vibronic spectroscopy in internal coordinates," J. Chem. Phys. 144, 084114 (2016).

${ }^{88}$ T. Yanai, D. P. Tew, and N. C. Handy, "A new hybrid exchangecorrelation functional using the Coulomb-attenuating method (CAM-B3LYP)," Chem. Phys. Lett. 393, 51-57 (2004).

${ }^{89}$ T. H. Dunning Jr., "Gaussian basis sets for use in correlated molecular calculations. i. the atoms boron through neon and hydrogen," J. Chem. Phys. 90, 1007 (1989).

${ }^{90}$ M. J. Frisch, G. W. Trucks, H. B. Schlegel, G. E. Scuseria, M. A. Robb, J. R. Cheeseman, G. Scalmani, V. Barone, G. A. Petersson, H. Nakatsuji, X. Li, M. Caricato, A. V. Marenich, J. Bloino, B. G. Janesko, R. Gomperts, B. Mennucci, H. P. Hratchian, J. V. Ortiz, A. F. Izmaylov, J. L. Sonnenberg, D. WilliamsYoung, F. Ding, F. Lipparini, F. Egidi, J. Goings, B. Peng, A. Petrone, T. Henderson, D. Ranasinghe, V. G. Zakrzewski, J. Gao, N. Rega, G. Zheng, W. Liang, M. Hada, M. Ehara, K. Toyota, R. Fukuda, J. Hasegawa, M. Ishida, T. Nakajima, Y. Honda, O. Kitao, H. Nakai, T. Vreven, K. Throssell, J. A. Montgomery, Jr., J. E. Peralta, F. Ogliaro, M. J. Bearpark, 
J. J. Heyd, E. N. Brothers, K. N. Kudin, V. N. Staroverov, T. A. Keith, R. Kobayashi, J. Normand, K. Raghavachari, A. P. Rendell, J. C. Burant, S. S. Iyengar, J. Tomasi, M. Cossi, J. M. Millam, M. Klene, C. Adamo, R. Cammi, J. W. Ochterski, R. L. Martin, K. Morokuma, O. Farkas, J. B. Foresman, and D. J. Fox, "Gaussian Development Version, Revision I.09," (2016), Gaussian Inc. Wallingford CT.

${ }^{91} \mathrm{~S}$. Hirata and M. Head-Gordon, "Time-dependent density functional theory within the Tamm-Dancoff approximation," Chem. Phys. Lett. 314, 291-299 (1999).

92 J. Tomasi, B. Mennucci, and R. Cammi, "Quantum mechanical continuum solvation models," Chem. Rev. 105, 2999-3094 (2005), pMID: 16092826.

${ }^{93}$ I. S. Ufimtsev and T. J. Martinez, "Quantum Chemistry on Graphical Processing Units. 3. Analytical Energy Gradients and First Principles Molecular Dynamics," J. Chem. Theory Comput. 5, 2619-2628 (2009).

${ }^{94}$ W. L. Jorgensen, J. Chandrasekhar, and J. D. Madura, "Comparison of simple potential functions for simulating liquid water," J. Chem. Phys. 79, 926 (1983).

${ }^{95}$ A. M. Rosnik and C. Curutchet, "Theoretical Characterization of the Spectral Density of the Water-Soluble Chlorophyll-Binding Protein from Combined Quantum Mechan-
ics/Molecular Mechanics Molecular Dynamics Simulations," J. Chem. Theory Comput. 11, 5826-5837 (2015).

${ }^{96}$ S. Chandrasekaran, M. Aghtar, S. Valleau, A. Aspuru-Guzik, and U. Kleinekathöfer, "Influence of Force Fields and Qauntum Chemistry Approach on Spectral Densities of BChl $a$ in Solution and in FMO Proteins," J. Phys. Chem. B 119, 9995-10004 (2015).

${ }^{97}$ C. W. Kim, J. W. Park, and Y. M. Rhee, "Effect of Chromophore Potential Model on the Description of Exciton-Phonon Interactions," J. Phys. Chem. Lett. 6, 2875-2880 (2015).

${ }^{98}$ O. Andreussi, I. G. Prandi, M. Canpetella, G. Prampolini, and B. Mennucci, "Classical Force Fields Tailored for QM applications: Is it Really a Feasible Strategy?" J. Chem. Theory Comput. 13, 4636-4648 (2017).

${ }^{99}$ M. M. Davis and H. B. Helzer, "Titrimetric and equilibrium studies using indicators related to nile blue a." Anal. Chem. 38, 451-461 (1966), https://doi.org/10.1021/ac60235a020.

${ }^{100}$ A. G. Pour, C. N. Lincoln, V. Perlík, F. Šanda, and J. Hauer, "Anharmonic vibrational effects in linear and two-dimensional electronic spectra," Phys. Chem. Chem. Phys. 19, 24752-24760 (2017). 
Supplementary material for "Nonlinear spectroscopy in the condensed phase: The role of Duschinsky rotations and third order cumulant contributions"

Tim J. Zuehlsdorff, ${ }^{1, \text { a) }}$ Hanbo Hong, ${ }^{1}$ Liang Shi, ${ }^{1,}$ b) and Christine M. Isborn ${ }^{1, c)}$ Chemistry and Chemical Biology, University of California Merced, Merced, California 95343, USA

(Dated: 13 May 2020)

a) Electronic mail: tzuehlsdorff@ucmerced.edu

b) Electronic mail: lshi4@ucmerced.edu

c) Electronic mail: cisborn@ucmerced.edu 


\section{CONTENTS}

I. Non-linear spectroscopy in the third order cumulant approximation 3

A. The 3rd order response function 3

B. Evaluating time-ordered integrals in the frequency domain 6

C. Non-linear response functions from purely classical correlation functions: The use of quantum correction factors (QCFs)

II. The Generalized Brownian Oscillator Model (GBOM) 8

A. The exact one- and two-time correlation functions 9

B. Classical one- and two-time correlation functions 10

C. Simplified model: No Duschinsky mode-mixing 11

III. The two-mode GBOM 12

A. The QCF and 2nd order cumulant 2DES 12

B. The effect of the Duschinsky rotation 13

IV. Nile red in benzene 16

A. Computational details for constructing the GBOM 16

$\begin{array}{ll}\text { V. Methylene blue } & 17\end{array}$

A. Computational details for the MD simulations 17

B. Computational details for constructing the GBOM 19

$\begin{array}{ll}\text { C. Solvent effects captured in MD } & 19\end{array}$

VI. Quantifying the amount of non-linear coupling in the GBOM 25

$\begin{array}{ll}\text { References } & 26\end{array}$ 


\section{NON-LINEAR SPECTROSCOPY IN THE THIRD ORDER CUMULANT APPROXIMATION}

\section{A. The 3rd order response function}

The full third order response function of the two-level electronic system can be constructed from the sum of four terms given by thermal averages of the transition dipole operator $\mu:^{1}$

$$
\begin{aligned}
& R_{1}\left(t_{3}, t_{2}, t_{1}\right)=\left\langle\mu_{01}\left(\tau_{1}\right) \mu_{10}\left(\tau_{2}\right) \mu_{01}\left(\tau_{3}\right) \mu_{10}(0) \rho_{g}\right\rangle \\
& R_{2}\left(t_{3}, t_{2}, t_{1}\right)=\left\langle\mu_{01}(0) \mu_{10}\left(\tau_{2}\right) \mu_{01}\left(\tau_{3}\right) \mu_{10}\left(\tau_{1}\right) \rho_{g}\right\rangle \\
& R_{3}\left(t_{3}, t_{2}, t_{1}\right)=\left\langle\mu_{01}(0) \mu_{10}\left(\tau_{1}\right) \mu_{01}\left(\tau_{3}\right) \mu_{10}\left(\tau_{2}\right) \rho_{g}\right\rangle \\
& R_{4}\left(t_{3}, t_{2}, t_{1}\right)=\left\langle\mu_{01}\left(\tau_{3}\right) \mu_{10}\left(\tau_{2}\right) \mu_{01}\left(\tau_{1}\right) \mu_{10}(0) \rho_{g}\right\rangle
\end{aligned}
$$

where $\rho_{g}$ is the equilibrium density matrix of the electronic ground state, $\tau_{3}=t_{1}+t_{2}+t_{3}$, $\tau_{2}=t_{1}+t_{2}$ and $\tau_{1}=t_{1}$.

We can define the energy gap operator $U$ of a system with arbitrary nuclear Hamiltonians $H_{g}$ and $H_{e}$ as $U=H_{e}-H_{g}$. Within the Condon approximation, ${ }^{2,3}$ the transition dipole operators can be expanded in terms of the fluctuations of the energy gap operator $\delta U=$ $H_{e}-H_{g}-\left\langle U \rho_{g}\right\rangle$ such that

$$
\begin{aligned}
\mu_{01}(t) & =|\mu| \exp _{+}\left[-\mathrm{i} \int_{0}^{t} \mathrm{~d} \tau \delta U(\tau)\right] \\
& =|\mu| \mathcal{T}_{+}\left[1-\mathrm{i} \int_{0}^{t} \delta U(\tau) \mathrm{d} \tau-\frac{1}{2} \int_{0}^{t} \mathrm{~d} \tau^{\prime \prime} \int_{0}^{t} \mathrm{~d} \tau^{\prime} \delta U\left(\tau^{\prime \prime}\right) \delta U\left(\tau^{\prime}\right) \ldots\right] \\
\mu_{10}(t) & =|\mu| \exp _{-}\left[+\mathrm{i} \int_{0}^{t} \mathrm{~d} \tau \delta U(\tau)\right] \\
& =|\mu| \mathcal{T}_{-}\left[1+\mathrm{i} \int_{0}^{t} \delta U(\tau) \mathrm{d} \tau-\frac{1}{2} \int_{0}^{t} \mathrm{~d} \tau^{\prime \prime} \int_{0}^{t} \mathrm{~d} \tau^{\prime} \delta U\left(\tau^{\prime \prime}\right) \delta U\left(\tau^{\prime}\right) \ldots\right]
\end{aligned}
$$

where $\exp _{+}$and $\exp _{-}$denote positively and negatively time-ordered integrals and $\mathcal{T}_{+}$and $\mathcal{T}_{-}$are the positive and negative time-ordering operators.

The terms $R_{1}-R_{4}$ can be evaluated by making use of a cumulant expansion of the energy gap fluctuation operator. ${ }^{1}$ In systems where the energy gap operator is only linearly dependent on nucleic positions, it can be shown that a cumulant expansion to second order is exact. For any non-linearly coupling of nuclear degrees of freedom to the energy gap, this is no longer the case and in principle an infinite number of cumulants have to be considered. We here focus on the deriving a general expression for the first correction term beyond the 
second order cumulant approximation, that is given by the third order cumulant. Under the third order cumulant approximation, the function $R_{1}$ can then be written in terms of a second order cumulant contribution $R_{1}^{\{2\}}$ and a third order cumulant correction $R_{1}^{\{3\}}$ such that

$$
R_{1}\left(t_{3}, t_{2}, t_{1}\right)=|\mu|^{4} R_{1}^{\{2\}}\left(t_{3}, t_{2}, t_{1}\right) R_{1}^{\{3\}}\left(t_{3}, t_{2}, t_{1}\right)
$$

with equivalent expressions for $R_{2}-R_{4}$.

The second order cumulant contribution $R^{\{2\}}$ is well known and can be expressed as ${ }^{1}$

$$
\begin{aligned}
R_{1}^{\{2\}}\left(t_{3}, t_{2}, t_{1}\right)= & \exp \left[-g_{2}\left(t_{1}\right)-g_{2}^{*}\left(t_{2}\right)-g_{2}^{*}\left(t_{3}\right)+g_{2}\left(t_{1}+t_{2}\right)\right. \\
& \left.+g_{2}^{*}\left(t_{2}+t_{3}\right)-g_{2}\left(t_{1}+t_{2}+t_{3}\right)\right], \\
R_{2}^{\{2\}}\left(t_{3}, t_{2}, t_{1}\right)= & \exp \left[-g_{2}^{*}\left(t_{1}\right)+g_{2}\left(t_{2}\right)-g_{2}^{*}\left(t_{3}\right)-g_{2}^{*}\left(t_{1}+t_{2}\right)\right. \\
& \left.-g_{2}\left(t_{2}+t_{3}\right)+g_{2}^{*}\left(t_{1}+t_{2}+t_{3}\right)\right], \\
R_{3}^{\{2\}}\left(t_{3}, t_{2}, t_{1}\right)= & \exp \left[-g_{2}^{*}\left(t_{1}\right)+g_{2}^{*}\left(t_{2}\right)-g_{2}\left(t_{3}\right)-g_{2}^{*}\left(t_{1}+t_{2}\right)\right. \\
& \left.-g_{2}^{*}\left(t_{2}+t_{3}\right)+g_{2}^{*}\left(t_{1}+t_{2}+t_{3}\right)\right], \\
R_{4}^{\{2\}}\left(t_{3}, t_{2}, t_{1}\right)= & \exp \left[-g_{2}\left(t_{1}\right)-g_{2}\left(t_{2}\right)-g_{2}\left(t_{3}\right)+g_{2}\left(t_{1}+t_{2}\right)\right. \\
& \left.+g_{2}\left(t_{2}+t_{3}\right)-g_{2}\left(t_{1}+t_{2}+t_{3}\right)\right] .
\end{aligned}
$$

Here, $g_{2}(t)$ is the second order lineshape function that can be expressed as a time-ordered integral of the quantum autocorrelation function of energy-gap fluctuations $C_{\delta U}^{\{2\}}(t)=$ $\left\langle\delta U(t) \delta U(0) \rho_{g}\right\rangle:$

$$
g_{2}(t)=\int_{0}^{t} \mathrm{~d} \tau^{\prime \prime} \int_{0}^{\tau^{\prime \prime}} \mathrm{d} \tau^{\prime} C_{\delta U}^{\{2\}}\left(\tau^{\prime \prime}-\tau^{\prime}\right)
$$

By expanding the transition dipole operators $\mu_{01}(t)$ and $\mu_{10}(t)$ to third order in $\delta U$ and collecting all terms involving three $\delta U$ operators, we can derive the equivalent expressions 
for $R^{\{3\}}$, yielding:

$$
\begin{aligned}
R_{1}^{\{3\}}\left(t_{1}, t_{2}, t_{3}\right)= & \exp \left[-g_{3}\left(t_{1}\right)-g_{3}^{*}\left(t_{1}+t_{2}\right)-g_{3}\left(t_{1}+t_{2}+t_{3}\right)\right. \\
& +h_{3}\left(t_{1}, t_{1}+t_{2}, t_{1}+t_{2}+t_{3}\right)+h_{1}\left(t_{1}, t_{1}+t_{2}\right)-h_{1}\left(t_{1}, t_{1}+t_{2}+t_{3}\right) \\
& -h_{2}\left(t_{1}+t_{2}, t_{1}+t_{2}+t_{3}\right)-h_{4}\left(t_{1}, t_{1}+t_{2}+t_{3}\right)+h_{4}\left(t_{1}+t_{2}, t_{1}+t_{2}+t_{3}\right) \\
& \left.-h_{5}\left(t_{1}, t_{1}+t_{2}\right)\right] \\
R_{2}^{\{3\}}\left(t_{1}, t_{2}, t_{3}\right)= & \exp \left[-g_{3}^{*}\left(t_{1}+t_{2}\right)-g_{3}\left(t_{1}+t_{2}+t_{3}\right)-g_{3}^{*}\left(t_{1}\right)\right. \\
& -h_{3}\left(t_{1}+t_{2}, t_{1}+t_{2}+t_{3}, t_{1}\right)-h_{2}\left(t_{1}+t_{2}, t_{1}+t_{2}+t_{3}\right)+h_{2}\left(t_{1}+t_{2}, t_{1}\right) \\
& +h_{1}\left(t_{1}+t_{2}+t_{3}, t_{1}\right)+h_{4}\left(t_{1}+t_{2}, t_{1}+t_{2}+t_{3}\right)+h_{5}\left(t_{1}+t_{2}, t_{1}\right) \\
& \left.-h_{5}\left(t_{1}+t_{2}+t_{3}, t_{1}\right)\right] \\
R_{3}^{\{3\}}\left(t_{1}, t_{2}, t_{3}\right)= & \exp \left[-g_{3}^{*}\left(t_{1}\right)-g_{3}\left(t_{1}+t_{2}+t_{3}\right)-g_{3}^{*}\left(t_{1}+t_{2}\right)\right. \\
& -h_{3}\left(t_{1}, t_{1}+t_{2}+t_{3}, t_{1}+t_{2}\right)-h_{2}\left(t_{1}, t_{1}+t_{2}+t_{3}\right)+h_{2}\left(t_{1}, t_{1}+t_{2}\right) \\
& +h_{1}\left(t_{1}+t_{2}+t_{3}, t_{1}+t_{2}\right)+h_{4}\left(t_{1}, t_{1}+t_{2}+t_{3}\right)+h_{5}\left(t_{1}, t_{1}+t_{2}\right) \\
& \left.-h_{5}\left(t_{1}+t_{2}+t_{3}, t_{1}+t_{2}\right)\right] \\
& \left.-h_{5}\left(t_{1}+t_{2}+t_{3}, t_{1}+t_{2}\right)\right] . \\
R_{4}^{\{3\}}\left(t_{1}, t_{2}, t_{3}\right)= & \exp \left[-g_{3}\left(t_{1}+t_{2}+t_{3}\right)-g_{3}^{*}\left(t_{1}+t_{2}\right)-g_{3}\left(t_{1}\right)\right. \\
& +h_{3}\left(t_{1}+t_{2}+t_{3}, t_{1}+t_{2}, t_{1}\right)+h_{1}\left(t_{1}+t_{2}+t_{3}, t_{1}+t_{2}\right)-h_{1}\left(t_{1}+t_{2}+t_{3}, t_{1}\right) \\
& -h_{2}\left(t_{1}+t_{2}, t_{1}\right)-h_{4}\left(t_{1}+t_{2}+t_{3}, t_{1}\right)+h_{4}\left(t_{1}+t_{2}, t_{1}\right) \\
&
\end{aligned}
$$

where the terms $h_{1}-h_{5}$ and $g_{3}$ can all be defined in terms of integrals over the two-time quantum correlation function of energy gap fluctuations $C_{\delta U}^{\{3\}}\left(t_{1}, t_{2}\right)=\left\langle\delta U\left(t_{2}\right) \delta U\left(t_{1}\right) \delta U(0) \rho_{g}\right\rangle$ : 


$$
\begin{aligned}
g_{3}(t) & =-\mathrm{i} \int_{0}^{t} \mathrm{~d} \tau^{\prime \prime} \int_{0}^{\tau^{\prime \prime}} \mathrm{d} \tau^{\prime} \int_{0}^{\tau^{\prime}} \mathrm{d} \tau C_{\delta U}^{\{3\}}\left(\tau^{\prime}-\tau, \tau^{\prime \prime}-\tau\right) \\
h_{1}\left(t_{1}, t_{2}\right) & =-\mathrm{i} \int_{0}^{t_{1}} \mathrm{~d} \tau^{\prime \prime} \int_{0}^{\tau^{\prime \prime}} \mathrm{d} \tau^{\prime} \int_{0}^{t_{2}} \mathrm{~d} \tau C_{\delta U}^{\{3\}}\left(\tau^{\prime}-\tau, \tau^{\prime \prime}-\tau\right) \\
h_{2}\left(t_{1}, t_{2}\right) & =-\mathrm{i} \int_{0}^{t_{1}} \mathrm{~d} \tau^{\prime} \int_{0}^{\tau^{\prime}} \mathrm{d} \tau^{\prime \prime} \int_{0}^{t_{2}} \mathrm{~d} \tau C_{\delta U}^{\{3\}}\left(\tau^{\prime}-\tau, \tau^{\prime \prime}-\tau\right) \\
h_{4}\left(t_{1}, t_{2}\right) & =-\mathrm{i} \int_{0}^{t_{1}} \mathrm{~d} \tau^{\prime \prime} \int_{0}^{t_{2}} \mathrm{~d} \tau^{\prime} \int_{0}^{\tau^{\prime}} \mathrm{d} \tau C_{\delta U}^{\{3\}}\left(\tau^{\prime}-\tau, \tau^{\prime \prime}-\tau\right) \\
h_{5}\left(t_{1}, t_{2}\right) & =-\mathrm{i} \int_{0}^{t_{1}} \mathrm{~d} \tau^{\prime \prime} \int_{0}^{t_{2}} \mathrm{~d} \tau \int_{0}^{\tau} \mathrm{d} \tau^{\prime} C_{\delta U}^{\{3\}}\left(\tau^{\prime}-\tau, \tau^{\prime \prime}-\tau\right) \\
h_{3}\left(t_{1}, t_{2}, t_{3}\right) & =-\mathrm{i} \int_{0}^{t_{1}} \mathrm{~d} \tau^{\prime \prime} \int_{0}^{t_{2}} \mathrm{~d} \tau^{\prime} \int_{0}^{t_{3}} \mathrm{~d} \tau C_{\delta U}^{\{3\}}\left(\tau^{\prime}-\tau, \tau^{\prime \prime}-\tau\right)
\end{aligned}
$$

The term $g_{3}(t)$ defines the third order cumulant correction to the lineshape function in linear spectroscopy, ${ }^{4,5}$ auxilliary functions $h_{1}, h_{2}, h_{4}$ and $h_{5}$ involve time-ordered integrals over two time variables, whereas $h_{3}$ is given by non-time-ordered integrals over the three time variables in $C_{\delta U}^{\{3\}}\left(\tau^{\prime}-\tau, \tau^{\prime \prime}-\tau\right)$.

\section{B. Evaluating time-ordered integrals in the frequency domain}

The integrals over time variables for the second and third order lineshape functions $g_{2}(t)$ and $g_{3}(t)$, as well as the auxilliary functions $h_{1}-h_{5}$ can be evaluated analytically by switching into Fourier space. We define the Fourier representation of the one- and two-time quantum correlation functions as

$$
\begin{aligned}
C_{\delta U}^{\{2\}}(\omega) & =\int \mathrm{d} t e^{\mathrm{i} \omega t} C_{\delta U}^{\{2\}}(t) \\
C_{\delta U}^{\{3\}}\left(\omega, \omega^{\prime}\right) & =\int \mathrm{d} t e^{\mathrm{i} \omega t} \int \mathrm{d} t^{\prime} e^{\mathrm{i} \omega^{\prime} t^{\prime}} C_{\delta U}^{(3)}\left(t, t^{\prime}\right)
\end{aligned}
$$

. The 2nd order lineshape function $g_{2}(t)$ can then be expressed as

$$
\begin{aligned}
g_{2}(t) & =\frac{1}{2 \pi} \int \mathrm{d} \omega \frac{C_{\delta U}^{(2)}(\omega)}{\omega^{2}}\left[1-e^{-\mathrm{i} \omega t}-\mathrm{i} \omega t\right] \\
& =\frac{1}{\pi} \int_{0}^{\infty} d \omega \frac{\mathcal{J}(\omega)}{\omega^{2}}\left[\operatorname{coth}\left(\frac{\beta \omega}{2}\right)[1-\cos (\omega t)]-\mathrm{i}[\sin (\omega t)-\omega t]\right]
\end{aligned}
$$

where $\mathcal{J}(\omega)$ is the so-called spectral density defined as the anti-symmetric part of the quantum auto-correlation function in the frequency domain:

$$
\mathcal{J}(\omega)=\mathrm{i} \theta(\omega) \int d t e^{\mathrm{i} \omega t} \operatorname{Im} C_{\delta U}(t)
$$


We have recently shown ${ }^{5}$ that the third order cumulant correction to the lineshape function $g_{3}(t)$ can be similarly written in terms of an integral over the frequency domain:

$$
g_{3}(t)=\int \mathrm{d} \omega_{1} \int \mathrm{d} \omega_{2} \frac{C_{\delta U}^{\{3\}}\left(\omega_{1}, \omega_{2}\right)}{4 \pi^{2} \bar{\omega} \omega_{1} \omega_{2}}\left[\mathrm{i} \omega_{1} t+\frac{\omega_{2}}{\bar{\omega}}\left(1-e^{-\mathrm{i} \bar{\omega} t}\right)-\left(1-e^{-\mathrm{i} \omega_{2} t}\right)\left(1+\frac{\omega_{1}}{\omega_{2}}\right)\right]
$$

where $\bar{\omega}=\omega_{1}+\omega_{2}$. The auxiliary terms $h_{1}-h_{5}$ necessary for constructing the full third order response function of the system in the third order cumulant approximation can be written in analogous terms, such that

$$
\begin{aligned}
h_{1}\left(t_{1}, t_{2}\right) & =\int \mathrm{d} \omega_{1} \int \mathrm{d} \omega_{2} \frac{C_{\delta U}^{\{3\}}\left(\omega_{1}, \omega_{2}\right)\left(1-e^{\mathrm{i} \bar{\omega} t_{2}}\right)}{4 \pi^{2} \bar{\omega} \omega_{1}}\left[\frac{1-e^{-\mathrm{i} \bar{\omega} t_{1}}}{\bar{\omega}}-\frac{1-e^{-\mathrm{i} \omega_{2} t_{1}}}{\omega_{2}}\right] \\
h_{2}\left(t_{1}, t_{2}\right) & =\int \mathrm{d} \omega_{1} \int \mathrm{d} \omega_{2} \frac{C_{\delta U}^{\{3\}}\left(\omega_{1}, \omega_{2}\right)\left(1-e^{\mathrm{i} \bar{\omega} t_{2}}\right)}{4 \pi^{2} \bar{\omega} \omega_{2}}\left[\frac{1-e^{-\mathrm{i} \bar{\omega} t_{1}}}{\bar{\omega}}-\frac{1-e^{-\mathrm{i} \omega_{1} t_{1}}}{\omega_{1}}\right] \\
h_{4}\left(t_{1}, t_{2}\right) & =-\int \mathrm{d} \omega_{1} \int \mathrm{d} \omega_{2} \frac{C_{\delta U}^{\{3\}}\left(\omega_{1}, \omega_{2}\right)\left(1-e^{-\mathrm{i} \omega_{2} t_{1}}\right)}{4 \pi^{2} \bar{\omega} \omega_{2}}\left[\frac{1-e^{-\mathrm{i} \omega_{1} t_{2}}}{\omega_{1}}+\frac{1-e^{\mathrm{i} \omega_{2} t_{2}}}{\omega_{2}}\right] \\
h_{5}\left(t_{1}, t_{2}\right) & =-\int \mathrm{d} \omega_{1} \int \mathrm{d} \omega_{2} \frac{C_{\delta U}^{\{3\}}\left(\omega_{1}, \omega_{2}\right)\left(1-e^{-\mathrm{i} \omega_{2} t_{1}}\right)}{4 \pi^{2} \omega_{1} \omega_{2}}\left[\frac{1-e^{\mathrm{i} \bar{\omega} t_{2}}}{\bar{\omega}}-\frac{1-e^{\mathrm{i} \omega_{2} t_{2}}}{\omega_{2}}\right] \\
h_{3}\left(t_{1}, t_{2}, t_{3}\right) & =-\int \mathrm{d} \omega_{1} \int \mathrm{d} \omega_{2} \frac{C_{\delta U}^{\{3\}}\left(\omega_{1}, \omega_{2}\right)\left(1-e^{\mathrm{i} \bar{\omega} t_{3}}\right)\left(1-e^{-\mathrm{i} \omega_{2} t_{1}}\right)\left(1-e^{-\mathrm{i} \omega_{1} t_{2}}\right)}{4 \pi^{2} \bar{\omega} \omega_{1} \omega_{2}}
\end{aligned}
$$

The above expressions are general and valid for any two-level electronic system within the Condon approximation. Thus if the one- and two-time quantum correlation functions are known, the non-linear response function under the third order cumulant approximation can be readily constructed.

\section{Non-linear response functions from purely classical correlation functions: The use of quantum correction factors (QCFs)}

The full quantum correlation functions $C_{\delta U}^{\{2\}}$ and $C_{\delta U}^{\{3\}}$ are generally not known in realistic systems, making it desirable to use approximate schemes to relate the quantum correlation functions to their classical counterparts, which are much easier to obtain. ${ }^{6-8}$

For the quantum autocorrelation function of energy-gap fluctuations, a standard approach to achieve this is through the use of the harmonic quantum correction factor, which can be derived by relating the quantum correlation function its Kubo-transformed counterpart that shares the same symmetries as the classical correlation function. ${ }^{9,10}$ Under this 
approximation one can write

$$
\begin{aligned}
C_{\delta U}^{(2)}(\omega) & \approx \frac{\beta \omega}{1-e^{-\beta \omega}} C_{\delta U}^{\{2\}, \mathrm{cl}}(\omega) \\
\mathcal{J}(\omega) & \approx \theta(\omega) \frac{\beta \omega}{2} C_{\delta U}^{\{2\}, \mathrm{cl}}(\omega) .
\end{aligned}
$$

where $\beta=1 / k_{\mathrm{B}} T$ and $\theta(\omega)$ is the Heaviside step function.

For the two-time quantum correlation function a similar approximate relationship has been derived recently, ${ }^{11}$ such that

$$
C_{\delta U}^{\{3\}}\left(\omega, \omega^{\prime}\right) \approx \frac{\bar{\omega} \omega^{\prime} \omega \beta^{2}}{2\left(\omega^{\prime} e^{-\beta \bar{\omega}}-\bar{\omega} e^{-\beta \omega^{\prime}}+\omega\right)} C_{\delta U}^{\{3\}, \mathrm{cl}}\left(\omega, \omega^{\prime}\right)
$$

Eqn. 27 has been successfully used to construct linear absorption spectra in the cumulant approach for moderately anharmonic systems. ${ }^{5}$ In this work, we extend the analysis to the construction of non-linear response functions. Using Eqn. 27 and Eqn. 26 it is then possible to construct the full nonlinear response function in the third order cumulant approximation using purely classical correlation functions as an input.

\section{THE GENERALIZED BROWNIAN OSCILLATOR MODEL (GBOM)}

The Generalized Brownian Oscillator Model (GBOM) is a simple model Hamiltonian for a two-level system, where the ground- and excited state potential energy surfaces are harmonic, such that

$$
\begin{aligned}
& H_{g}\left(\hat{\mathbf{q}}_{g}, \hat{\mathbf{p}}_{g}\right)=\frac{1}{2} \sum_{j}\left[\hat{p}_{g, j}^{2}+\omega_{g, j}^{2} \hat{q}_{g, j}^{2}\right], \\
& H_{e}\left(\hat{\mathbf{q}}_{e}, \hat{\mathbf{q}}_{e}\right)=\frac{1}{2} \sum_{j}\left[\hat{p}_{e, j}^{2}+\omega_{e, j}^{2} \hat{q}_{e, j}^{2}\right]+\omega_{e g}^{0},
\end{aligned}
$$

where $\omega_{g, j}$ and $\omega_{e, j}$ are the frequencies of the ground and excited state normal modes and the coordinates of the normal modes on the ground and excited state PESs can be connected via a linear transformation called the Duschinsky rotation matrix, J,

$$
\hat{q}_{g, n}=\sum_{m} J_{n m} \hat{q}_{e, m}+K_{n}
$$

$K_{n}$ is the shift vector that denotes the displacement between the minima of the ground- and excited state potential energy surface. 
The GBOM is a convenient model Hamiltonian for studying optical properties of complex systems, as its simple form allows for the derivation of analytical expressions for the exact one- and two-time quantum correlation functions. Furthermore, a Hamiltonian of this form can be obtained by performing a geometry optimization and normal mode analysis of a molecule in its ground- and excited state and can therefore be constructed using a range of standard quantum chemistry software packages. The model system did form the focus of a recent study regarding the performance of the cumulant approach in the context of linear response. ${ }^{5}$ Here we provide a short summary of the analytical expressions for the one- and two-time correlation functions derived in the previous work.

\section{A. The exact one- and two-time correlation functions}

For the GBOM, the energy gap operator as a function of ground state nuclear positions $\hat{\boldsymbol{q}}_{g}$ can be written as

$$
\delta U\left(\hat{\mathbf{q}}_{g}\right)=\sum_{n m} \Omega_{n m}^{2} \hat{q}_{g, n} \hat{q}_{g, m}-\sum_{n} \Gamma_{n} \hat{q}_{g, n}-\xi
$$

where

$$
\begin{aligned}
\Omega_{n m}^{2} & =\frac{1}{2}\left[\sum_{j} J_{n j}\left(\omega_{e, j}\right)^{2} J_{j m}^{T}-\delta_{n m}\left(\omega_{g, n}\right)^{2}\right] \\
\Gamma_{n} & =\sum_{j m} K_{m} J_{m j}\left(\omega_{e, j}\right)^{2} J_{j n}^{T} \\
\xi & =\frac{1}{2} \sum_{j} \frac{\Omega_{j j}^{2}}{\omega_{g, j}} \operatorname{coth}\left(\frac{\beta \omega_{g, j}}{2}\right)
\end{aligned}
$$

and the thermal average of the energy gap, $\omega_{e g}^{\text {av }}=\left\langle\rho_{g} U(\hat{\boldsymbol{q}})\right\rangle$, is given by

$$
\begin{aligned}
\omega_{e g}^{\mathrm{av}} & =\omega_{e g}^{0}+\xi+\lambda_{0} \\
\lambda_{0} & =\frac{1}{2} \sum_{j n m} K_{m} J_{m j}\left(\omega_{e, j}\right)^{2} J_{j n}^{T} K_{n} .
\end{aligned}
$$

The exact quantum mechanical energy gap autocorrelation function can then be written as

$$
\begin{aligned}
C_{\delta U}^{\{2\}}(t)=\sum_{i} & \frac{\Gamma_{i}^{2}}{2 \omega_{g, i}}\left[m_{i} e^{-\mathrm{i} \omega_{g, i} t}+n_{i} e^{\mathrm{i} \omega_{g, i} t}\right] \\
& +\sum_{i j} \frac{\left[\Omega_{i j}^{2}\right]^{2}}{2 \omega_{g, i} \omega_{g, j}}\left[m_{i} m_{j} e^{-\mathrm{i} \omega_{i j}^{+} t}+n_{i} n_{j} e^{\mathrm{i} \omega_{i j}^{+} t}+m_{i} n_{j} e^{-\mathrm{i} \omega_{i j}^{-} t}+n_{i} m_{j} e^{\mathrm{i} \omega_{i j}^{-} t}\right],
\end{aligned}
$$


where $n_{j}=n\left(\omega_{g, j}\right)=\left[e^{\beta \omega_{g, j}}-1\right]^{-1}$ is the average phonon number for the $j$ th mode, $m_{j}=n_{j}+1, \omega_{i j}^{+}=\omega_{g, i}+\omega_{g, j}$ and $\omega_{i j}^{-}=\omega_{g, i}-\omega_{g, j}$. We note that Eqn. 36 was derived in the SI of Ref. 5, but the final expression (corresponding to Eqn. S29 in the previous work) contained a typo. The expression presented here in Eqn. 36 is the correct version. Similarly, the two-time autocorrelation function can be computed as

$$
\begin{aligned}
C_{\delta U}^{\{3\}}\left(t_{1}, t_{2}\right)=\sum_{i j} & \frac{\Omega_{i j}^{2} \Gamma_{i} \Gamma_{j}}{2 \omega_{g, i} \omega_{g, j}}\left[m_{i} m_{j}\left(e^{-\mathrm{i}\left(\omega_{g, i} t_{2}+\omega_{g, j} t_{1}\right)}+e^{-\mathrm{i}\left(\omega_{g, i} t_{2}-\omega_{i j}^{-} t_{1}\right)}+e^{-\mathrm{i}\left(\omega_{i j}^{+} t_{2}-\omega_{g, i} t_{1}\right)}\right)\right. \\
& +n_{i} n_{j}\left(e^{\mathrm{i}\left(\omega_{g, i} t_{2}+\omega_{g, j} t_{1}\right)}+e^{\mathrm{i}\left(\omega_{i j}^{+} t_{2}-\omega_{g, i} t_{1}\right)}+e^{\mathrm{i}\left(\omega_{g, i} t_{2}-\omega_{i j}^{-} t_{1}\right)}\right) \\
& +m_{i} n_{j}\left(e^{-\mathrm{i}\left(\omega_{g, i} t_{2}-\omega_{g, j} t_{1}\right)}+e^{-\mathrm{i}\left(\omega_{g, i} t_{2}-\omega_{i j}^{+} t_{1}\right)}+e^{-\mathrm{i}\left(\omega_{i j}^{-} t_{2}-\omega_{g, i} t_{1}\right)}\right) \\
& \left.+n_{i} m_{j}\left(e^{\mathrm{i}\left(\omega_{g, i} t_{2}-\omega_{g, j} t_{1}\right)}+e^{\mathrm{i}\left(\omega_{g, i} t_{2}-\omega_{i j}^{+} t_{1}\right)}+e^{\mathrm{i}\left(\omega_{i j}^{-} t_{2}-\omega_{g, i} t_{1}\right)}\right)\right] \\
& +\sum_{i j k} \frac{\Omega_{i j}^{2} \Omega_{i k}^{2} \Omega_{j k}^{2}}{\omega_{g, i} \omega_{g, j} \omega_{g, k}}\left[m_{i} m_{j} m_{k} e^{-\mathrm{i}\left(\omega_{i j}^{+} t_{2}-\omega_{i k}^{-} t_{1}\right)}+n_{i} n_{j} n_{k} e^{\mathrm{i}\left(\omega_{i j}^{+} t_{2}-\omega_{i k}^{-} t_{1}\right)}\right. \\
& +m_{i} n_{j} m_{k} e^{-\mathrm{i}\left(\omega_{i j}^{-} t_{2}-\omega_{i k}^{-} t_{1}\right)}+n_{i} m_{j} n_{k} e^{\mathrm{i}\left(\omega_{i j}^{-} t_{2}-\omega_{i k}^{-} t_{1}\right)}+m_{i} n_{j} n_{k} e^{-\mathrm{i}\left(\omega_{i j}^{-} t_{2}-\omega_{i k}^{+} t_{1}\right)} \\
& \left.+n_{i} m_{j} m_{k} e^{\mathrm{i}\left(\omega_{i j}^{-} t_{2}-\omega_{i k}^{+} t_{1}\right)}+m_{i} m_{j} n_{k} e^{-\mathrm{i}\left(\omega_{i j}^{+} t_{2}-\omega_{i k}^{+} t_{1}\right)}+n_{i} n_{j} m_{k} e^{\mathrm{i}\left(\omega_{i j}^{+} t_{2}-\omega_{i k}^{+} t_{1}\right)}\right] .
\end{aligned}
$$

Note that the Fourier representations of $C_{\delta U}^{\{2\}}$ and $C_{\delta U}^{\{3\}}$ reduce to a sum of Dirac $\delta$ functions in frequency space, such that all quantities $g_{2}(t), g_{3}(t), h_{1}-h_{5}$ necessary to construct the full nonlinear response function of the GBOM in the third order cumulant approximation have closed-form expressions.

\section{B. Classical one- and two-time correlation functions}

To test the performance of the quantum correction factors introduced in Sec. IC it is advantageous to derive analytic expressions for the purely classical correlation functions $C_{\delta U}^{\{2\}, \mathrm{cl}}$ and $C_{\delta U}^{\{3\}, \mathrm{cl}}$ for the GBOM, where the exact quantum correlation functions are known. This can be done by taking the high-temperature limit of the quantum correlation functions in Eqn. 36 and 37, yielding

$$
C_{\delta U}^{\{2\}, \mathrm{cl}}(t)=\sum_{i j}\left(\frac{\Omega_{i j}^{2}}{\beta \omega_{g, i} \omega_{g, j}}\right)^{2}\left\{\cos \left(\omega_{i j}^{+} t\right)+\cos \left(\omega_{i j}^{-} t\right)\right\}+\sum_{i} \frac{\Gamma_{i}^{2}}{\beta \omega_{g, i}^{2}} \cos \left(\omega_{g, i} t\right),
$$


and

$$
\begin{aligned}
C_{\delta U}^{\{3\}, \mathrm{cl}}\left(t_{1}, t_{2}\right)=8 & \sum_{i j k} \frac{\Omega_{i j}^{2} \Omega_{i k}^{2} \Omega_{j k}^{2}}{\beta^{3}\left(\omega_{g, i} \omega_{g, j} \omega_{g, k}\right)^{2}} \cos \left(\omega_{g, i}\left(t_{2}-t_{1}\right)\right) \cos \left(\omega_{g, j} t_{2}\right) \cos \left(\omega_{g, k} t_{1}\right) \\
& +2 \sum_{i j} \frac{\Omega_{i j}^{2} \Gamma_{i} \Gamma_{j}}{\beta^{2}\left(\omega_{g, i} \omega_{g, j}\right)^{2}}\left\{\cos \left(\omega_{g, i}\left(t_{2}-t_{1}\right)\right) \cos \left(\omega_{g, j} t_{2}\right)\right. \\
& \left.\quad+\cos \left(\omega_{g, i}\left(t_{2}-t_{1}\right)\right) \cos \left(\omega_{g, j} t_{1}\right)+\cos \left(\omega_{g, i} t_{1}\right) \cos \left(\omega_{g, j} t_{2}\right)\right\} .
\end{aligned}
$$

We note that Eqn. 38 was derived in the SI of Ref. 5, but the final expression (corresponding to Eqn. S33 in the previous work) contained a typo. The expression presented here in Eqn. 36 is the correct version. Since the Fourier representations of the classical correlation functions for the GBOM reduce to the simple form of sums of Dirac $\delta$-functions, the approximate lineshape functions $\tilde{g}_{2}(t)$ and $\tilde{g}_{3}(t)$, as well as $\tilde{h}_{1}-\tilde{h}_{5}$ constructed using the quantum correction factors defined in Sec. I C have closed-form expressions.

\section{Simplified model: No Duschinsky mode-mixing}

The complexity of the GBOM can be significantly reduced by ignoring the effects of Duschinsky mode mixing that couples all vibrational modes in the system. This can be achieved by setting the Duschinsky matrix $\mathbf{J}=\mathbf{I}$, where $\mathbf{I}$ is the identity matrix. From this it follows that $\Omega_{n m}^{2}=\delta_{n m} \Omega_{m m}^{2}$. It is then straightforward to show that the exact oneand two-time correlation functions reduce to the expressions derived by Fidler et al. ${ }^{4}$ for a one-mode model system where ground- and excited state harmonic surfaces have different curvatures.

Furthermore, for this simplified form of the GBOM, one can also show that $h_{1}\left(t_{1}, t_{2}\right)=$ $h_{5}^{*}\left(t_{2}, t_{1}\right)$ and $h_{2}\left(t_{1}, t_{2}\right)=h_{4}^{*}\left(t_{2}, t_{1}\right)$ such that the third order correction to the nonlinear

response function derived in this work reduces to exactly the same form as reported in Ref. 4. It is worth noting however that this simplification does not hold for arbitrary quantum correlation functions $C_{\delta U}^{\{3\}}$ and does not hold for a GBOM with Duschinsky mode mixing. The expressions presented in Sec. I A are more general and hold for arbitrary nuclear Hamiltonians. 
a)

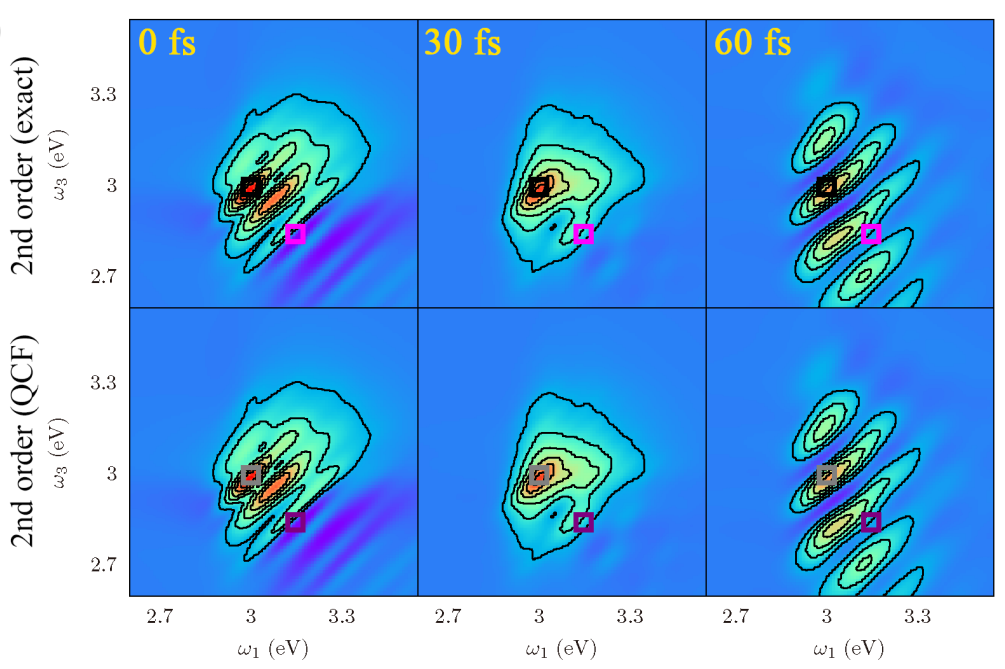

b)

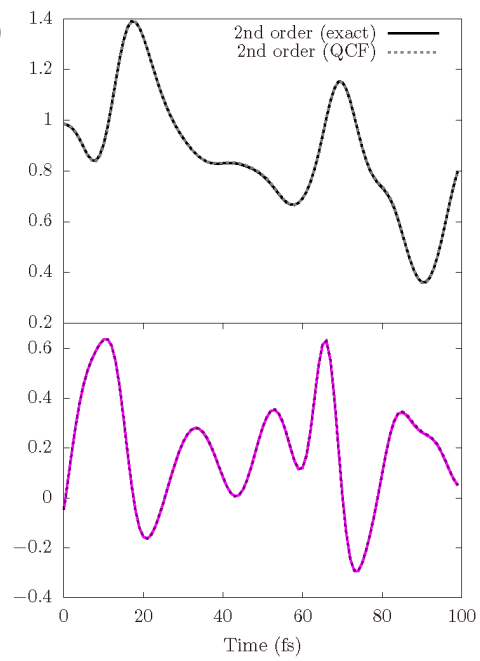

FIG. 1. Results for a two mode model system with a Duschinsky rotation angle of $10^{\circ}$ and an effective solvent broadening of $\lambda_{\text {solv }}=0.0022$ Ha: a) 2 DES results for $t_{\text {delay }}=0,30$ and 60 fs. The first row corresponds to second order cumulant results using the exact quantum correlation function and the second row shows the second order cumulant data constructed from the purely classical correlation function using QCFs; b) Time-evolution of integrated intensities for the two boxes shown in the 2DES spectra. Solid lines correspond to exact second order cumulant and dotted lines correspond to approximate second order cumulant results using QCFs.

\section{THE TWO-MODE GBOM}

\section{A. The QCF and 2nd order cumulant 2DES}

In the main text, we assess the accuracy of the choice of QCF in reproducing third order cumulant 2DES spectra from purely classical input. Here, we do a similar comparison for the second order cumulant approximation by considering the 2-mode model system with Duschinsky mode mixing discussed in the main text. A comparison of the resulting 2DES with three different delay times, as well as the time-evolution of integrated intensities for two points in the spectra, can be found in Fig. 1.

In close agreement with the results found for the linear spectra, the use of the harmonic QCF yields 2D electronic spectra that are indistinguishable from those constructed using the exact quantum correlation function for this two mode GBOM. The good agreement carries over to the time-evolution of integrated intensities, which is again virtually indistinguish- 
able for the exact quantum correlation function and the approximate correlation function constructed from purely classical input.

These results mirror the conclusions drawn in our previous study, ${ }^{5}$ where it was found that for the GBOM model Hamiltonian with moderate nonlinear coupling in the energy gap fluctuations, the second order cumulant linear absorption spectrum could be reproduced exactly using classical correlation functions and the harmonic QCF. This close agreement can be ascribed to the form of the exact and the approximately reconstructed quantum autocorrelation function for this system. $C_{\delta U}^{\{2\}}(t)$ consists of two terms (See Eqn. 36): A onephonon contribution of ground state frequencies and two-phonon contribution consistent of all possible combinations of two ground state frequencies. The harmonic QCF exactly reconstructs the correct one-phonon contribution from the classical autocorrelation function and only introduces errors in the two-phonon contribution. ${ }^{5}$ Since for GBOM parameters chosen to represent weakly to moderately nonlinear coupling in the energy gap fluctuations, the one-phonon term is dominant, it is not surprising that the 2DES signals in the second order cumulant approximation for the GBOM system can be almost exactly reproduced using classical correlation functions only.

We conclude that for a system whose PES closely matches that of a GBOM, the 2DES signal in the second order cumulant approximation can be accurately reconstructed using purely classical correlation functions as an input. For the third order cumulant contribution, the QCF introduced in Ref. ${ }^{11}$ does not reproduce any terms in $C_{\delta U}^{\{3\}}\left(t_{1}, t_{2}\right)$ exactly, even for the simplified GBOM. ${ }^{5}$ For this reason, discrepancies between third order cumulant nonlinear spectra based on exact quantum correlation functions and those based on purely classical input are expected to be larger, matching the results obtained in the main text of this work.

\section{B. The effect of the Duschinsky rotation}

In the main manuscript, we analyze the 2DES signals for a 2-mode GBOM model system with a Duschinksy mode-mixing of $10^{\circ}$. Both the Duschinsky rotation and the mismatch between ground- and excited state frequencies causes non-Gaussian energy-gap fluctuations, resulting in the second order cumulant approximation to no longer hold exactly. To isolate the non-Gaussian energy gap fluctuations introduced by the mode-mixing from the nonGaussian fluctuations due to the mismatch between ground- and excited state frequencies, 
a)

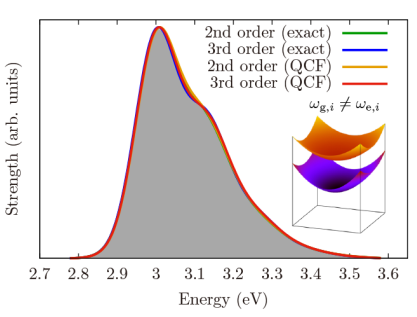

c)

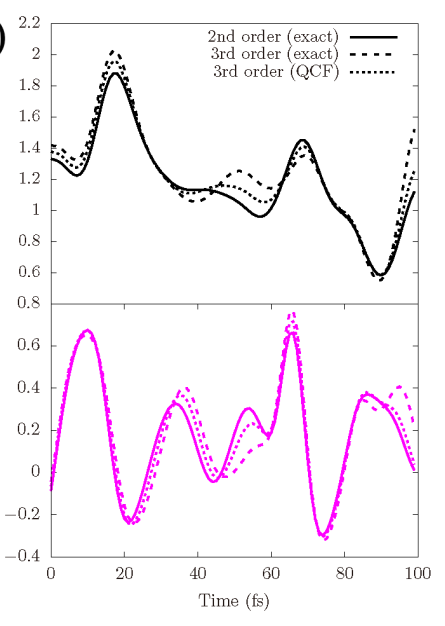

b)

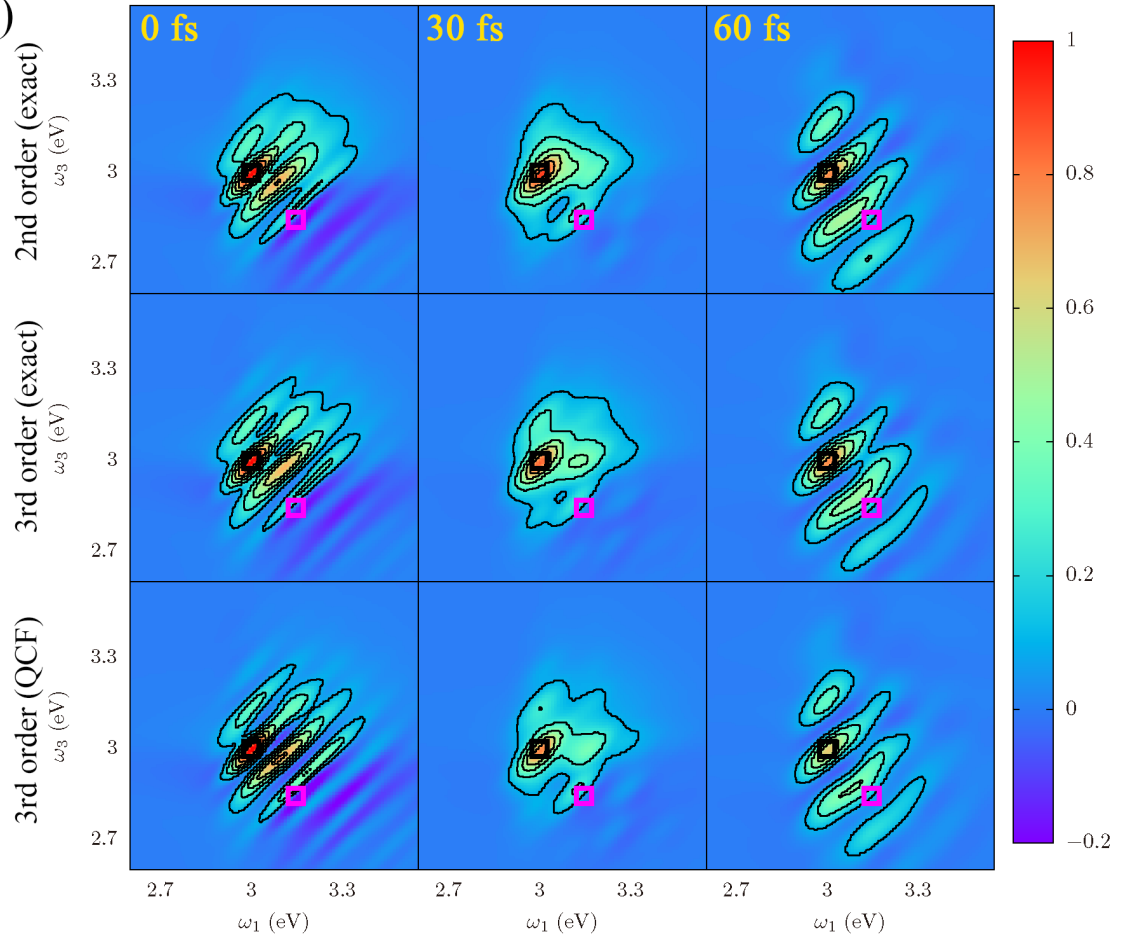

FIG. 2. Results for a two mode model system with a Duschinsky rotation angle of $0^{\circ}$ and an effective solvent broadening of $\lambda_{\text {solv }}=0.0022 \mathrm{Ha}$ : a) Linear spectra in the cumulant approach in comparison with the exact spectrum (shown in gray); b) 2DES results for $t_{\text {delay }}=0,30$ and 60 fs. The first row corresponds to second order cumulant results using the exact quantum correlation function, the second row contains the third order cumulant data for the exact quantum correlation function and the third row shows the third order cumulant data constructed from the purely classical correlation function using QCFs; c) Time-evolution of integrated intensities for the two boxes shown in the 2DES spectra. Solid lines correspond to exact second order cumulant, dashed lines show exact third order cumulant and dotted lines correspond to approximate third order cumulant results using QCFs.

we repeat the same calculations presented in the main manuscript, but where the Duschinsky matrix $\mathbf{J}=\mathbf{I}$ with $\mathbf{I}$ being the identity matrix. The resulting linear and nonlinear spectra for this system, containing only mismatching ground- and excited state frequencies but no mode mixing, in the second and third order cumulant approach can be found in Fig. 2 .

From Fig. 2 a) it becomes clear that switching off the Duschinksy mode mixing significantly reduces the size of the third order cumulant correction in the linear spectrum. In 
contrast to the full system with Duschinsky mode mixing presented in the main text, the second order cumulant linear absorption spectra are in relatively good agreement with both the exact spetrum and the third order cumulant spectra, both for the exact correlation functions and the approximate correlation functions constructed from QCFs. In contrast, the 2DES signal (Fig. 2 b)) does show some significant differences between second order and third order cumulant, even when Duschinsky mode-mixing is suppressed. Similarly to the results presented in the main text, some vibronic features are sharpened in the third order cumulant approximation. This is also evident when looking at the time-evolution of integrated intensities shown in Fig. 2 c), where the third order cumulant corrections lead to more pronounced oscillations and slight shifts in peaks. As in the results for the main manuscript, the approximate third order cumulant spectra constructed from purely classical input do not fully capture the exact third order cumulant results, but represent a clear improvement over the second order cumulant results.

To further analyze the differences between the two-mode model systems with and with without Duschinksy mode mixing, Fig. 3 shows the time-evolution of integrated intensities based on the exact quantum correlation functions for both systems. As can be seen, in the second order cumulant approximation there are only minor differences between the time evolution of the intensities for the fully coupled system and the system where $\mathbf{J}=\mathbf{I}$. The most significant change can be observed for the vibronic feature corresponding to $\omega_{1}=\omega_{3}=$ $E_{0-0}$, with $E_{0-0}$ being the energy of the 0-0 transition of the system. Here, the Duschinsky mode mixing causes an overall drop in intensity in this feature, that is almost constant over the 100 fs time period probed.

While the second order cumulant signals for the system with and without Duschinsky rotation are fairly similar, the same is not true for the third order cumulant signals. Here, the third order cumulant correction to the model system without mode mixing is considerably smaller than the third order cumulant correction in the fully coupled system. 


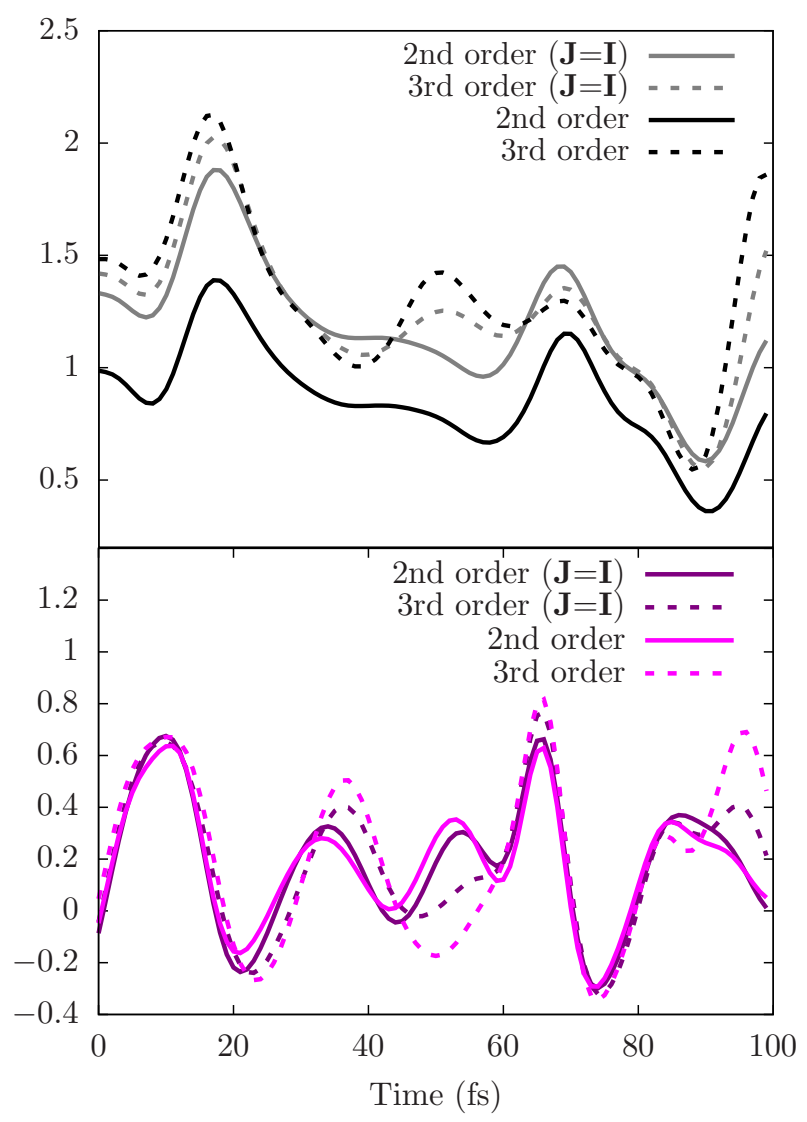

FIG. 3. Time-evolution of integrated intensities of the two mode model system, both with and without Duschinsky mode-mixing, for the two boxes shown in the 2DES spectra in the main manuscript. Solid lines correspond to exact 2nd order cumulant, dashed lines show exact 3rd order cumulant.

\section{NILE RED IN BENZENE}

\section{A. Computational details for constructing the GBOM}

To construct an effective GBOM for the Nile red molecule in benzene, ground- and excited state geometry optimizations and normal mode calculations were performed in Gaussian ${ }^{12}$ using density-functional theory (DFT). The system was simulated at the CAM-B3LYP ${ }^{13} / 6$ $31+\mathrm{G}^{* 14}$ level of theory and the solvent environment was represented by a polarizable continuum model (PCM). ${ }^{15,16}$ For the excited state, we used time-dependent DFT (TDDFT) in the Tamm-Dancoff ${ }^{17}$ approximation and a non-equilibrium solvation approach. The shift vector $\mathbf{K}$ and the Duschinsky rotation matrix $\mathbf{J}$ where directly extracted from Gaussian 
using the functionality to compute Franck-Condon spectra. ${ }^{18,19}$

Solvent effects in the linear and nonlinear spectra were accounted for through a spectral density of the Debye form:

$$
\mathcal{J}_{\text {solv }}(\omega)=2 \lambda_{\text {solv }} \omega_{c} \frac{\omega}{\omega_{c}^{2}+\omega^{2}}
$$

where $\lambda_{\text {solv }}$ is the solvent reorganization energy and the cutoff frequency $\omega_{c}$ determines the time-scale of solvent relaxation. Importantly, it is assumed that the collective solvent effect can be described by a bath of brownian oscillators. This means that the effect of the solvent environment can be evaluated exactly in the second order cumulant approach, without any higher order cumulant contributions in either the linear or nonlinear spectra.

For the purpose of this work, we kept $\omega_{c}$ fixed to $\omega_{c}=22 \mathrm{~cm}^{-1}$, a value chosen to be smaller than any frequency defining the GBOM of the chromophore degrees of freedom. The reorganization energy $\lambda_{\text {solv }}$ was then chosen such that the width of the linear absorption spectrum as evaluated in a Franck-Condon approach ${ }^{20}$ matches the experimental absorption spectrum of Nile red in benzene, ${ }^{21}$ resulting in $\lambda_{\text {solv }}=0.095 \mathrm{eV}$.

\section{METHYLENE BLUE}

\section{A. Computational details for the MD simulations}

Methylene blue was simulated in water with force-field (FF) based molecular-dynamics (MD) using OpenMM. ${ }^{22}$ The TIP3P ${ }^{23} \mathrm{FF}$ was used for the water molecules and for Methylene blue a bespoke FF was constructed using the QUBEKit ${ }^{24}$ package based on ground state electronic structure calculations of the chromophore in vacuum at the CAM-B3LYP/6$31+\mathrm{G}^{*}$ level of theory using Gaussian. Methylene blue was solvated in a box containing 7068 TIP3P water molecules and a single $\mathrm{Cl}^{-}$counterion to balance the positive charge on the chromophore, and a 500 ps pressure equilibration in the NPT ensemble was performed. This was followed by a 6 ns production run in the NVT ensemble at $300 \mathrm{~K}$ using a Langevin ther-

mostat with a collision frequency of $1 \mathrm{ps}^{-1}$. An MD time-step of 1 fs was used throughout. Positions and velocities were extracted every $1 \mathrm{~ns}$, yielding 6 independent initial configurations for QM/MM simulations.

From the 6 independent initial configurations generated with FF based MD, we generated $6 \mathrm{QM} / \mathrm{MM}$ trajectories of 10 ps length using the inbuilt QM/MM functionality in the Ter- 
achem code. ${ }^{25}$ Since Terachem does not apply periodic boundary conditions, we carved out a sphere of $30 \AA$ radius around the chromophore center of mass, and all water molecules with centres of mass within that sphere were included in the QM/MM calculation. To keep the density of the water droplet constant during the simulation, we applied a spherical confining potential. While the process of changing from periodic boundary conditions to a spherical droplet in a harmonic confining potential for the QM/MM simulation is expected to disrupt the dynamics of the outer shell of water molecules, we expect this change in boundary conditions to have only minor effects on the chromophore and its 1st solvation shell at the center of the droplet on the timescale of the QM/MM simulation. The chromophore and its counter ion were treated quantum mechanically at the CAM-B3LYP/6-31+G* level of theory and all water molecules within the sphere were treated through the TIP3P force field. A MD time-step of 0.5 fs was used for the QM/MM simulation and the temperature was kept at $300 \mathrm{~K}$ using a Langevin thermostat with a collision frequency of $1 \mathrm{ps}^{-1}$. The first 2 ps of each QM/MM trajectory was discarded to allow the system to equilibrate after the switching between a pure MM and a QM/MM treatment, yielding 8 ps of usable dynamics for each trajectory. Snapshots were extracted every 2 fs, yielding 4000 snapshots per trajectory and a total of 24.000 snapshots.

For each of the 24.000 snapshots, we then computed vertical excitation energies using TDDFT in the Tamm-Dancoff approximation as implemented in the Terachem code. ${ }^{26}$ To account for polarization effects from first principles, ${ }^{5}$ an effective QM region including solvent environment was created by placing a sphere of $6 \AA$ radius on each chromophore molecule. Each solvent molecule with a centre of mass within the volume generated by the overlapping spheres was included in the calculation at the QM level, yielding $\approx 380$ atoms in the $\mathrm{QM}$ region. All other water molecules were included in the calculation in terms of classical point charges definde by the TIP3P water model. All TDDFT calculations were performed at the CAM-B3LYP/6-31+G* level of theory and the Tamm-Dancoff approximation was used throughout.

For all GBOMs discussed in this work, a spectral density of the Debye type is used to represent the low-frequency solvent degrees of freedom. Since the solvent degrees of freedom are represented by an infinite bath of displaced harmonic oscillators, they only contribute to the second order cumulant terms in the linear- and nonlinear spectroscopy experiments modelled in this work. To prevent the low frequency vibrational motions, that in any case 
are insufficiently sampled by the short QM/MM trajectories used in this work, to contribute to the third order cumulant correction in the MD-based model of Methylene blue, we apply

a filter to the classical two-time correlation function $C_{\delta U}^{\{3\}, \mathrm{cl}}$. This is achieved by setting $C_{\delta U}^{\{3\}, \mathrm{cl}}\left(\omega, \omega^{\prime}\right)=0$ for $\omega, \omega^{\prime}<200 \mathrm{~cm}^{-1}$. In this way, similarly to the GBOM calculations, the third order cumulant correction in the MD-based model is only due to high frequency 'chromophore' degrees of freedom and not due to the bath of low frequency solvent modes.

\section{B. Computational details for constructing the GBOM}

To construct an effective GBOM for Methylene blue similar steps as for Nile red in benzene are followed. Geometry optimizations and frequency calculations were carried out at the CAM-B3LYP/6-31+G* level of theory and the Tamm-Dancoff approximation was used throughout. However, unlike for Nile red in benzene, the system was not simulated in a PCM but in vacuum. This was done because the GBOM parameterized from the PCM calculation lead to divergent behaviour in the third order cumulant approach for the exact quantum correlation function, most likely due to some large nonlinear couplings for specific vibrational modes. Since the PCM in any case cannot capture direct solute-solvent interactions such as hydrogen bonding, which is likely important in this system, and since the second and third order cumulant lineshapes for the GBOM parameterized in vacuum closely match the cumulant lineshapes generated from MD, we use the GBOM parameterized in vacuum throughout this work.

To include solvent effects in the GBOM, we again add an infinite bath of solvent degrees of freedom following a Debye-type spectral density. As for Nile red in benzene, the characteristic cutoff frequency was fixed to $\omega_{c}=22 \mathrm{~cm}^{-1}$. The solvent reorganization energy $\lambda_{\text {solv }}$ was then chosen such that the second order cumulant spectrum based on the classical correlation function and the harmonic QCF computed for the GBOM agreed in width with the second order cumulant spectrum generated directly from MD, yielding $\lambda_{\text {solv }}=0.011 \mathrm{eV}$.

\section{Solvent effects captured in MD}

Generating cumulant spectra directly from correlation functions extracted from MD has some advantages over the computationally cheaper GBOM treatment, especially since it 


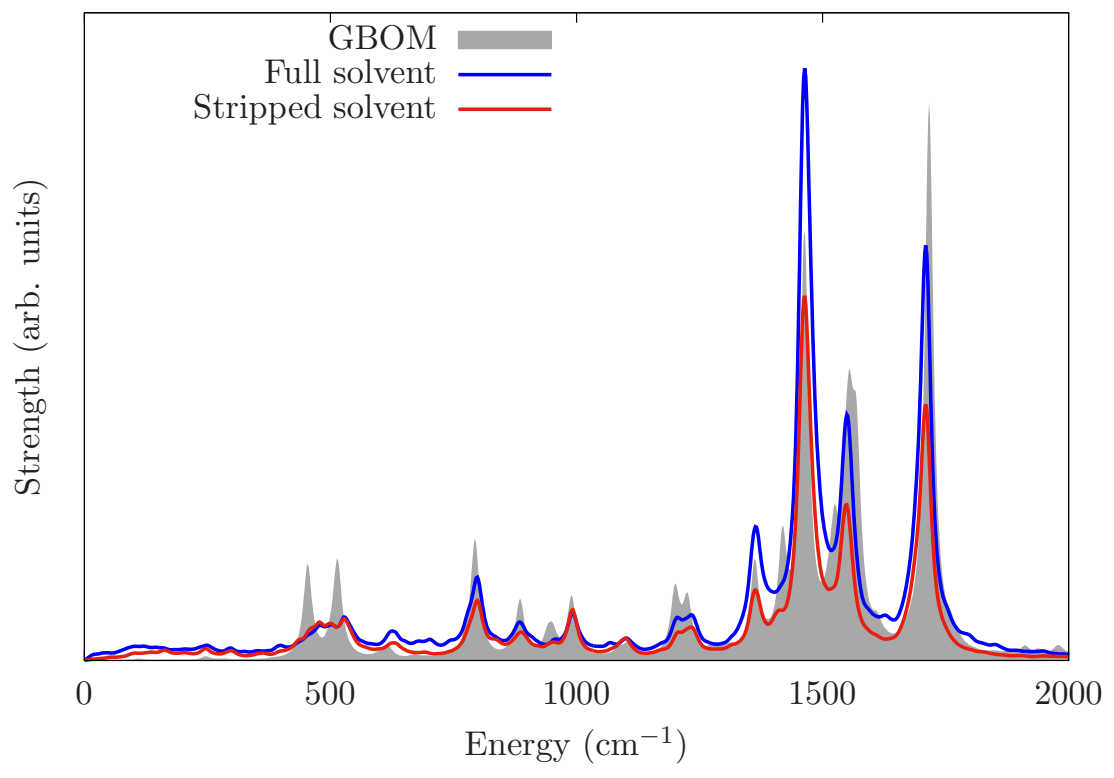

FIG. 4. Spectral density for a single 8 ps MD trajectory of Methylene blue, once where the vertical excitation energies are computed within $6 \AA$ QM regions of explicit water molecules, and once where the solvent environment is stripped from all snapshots. The spectral density for the GBOM is shown in gray.

allows for capturing direct couplings between the chromophore and its solvent environment. Here, we analyse the direct solvent effects on Methylene blue in more detail. To do so, we take the first 8 ps MD trajectory of Methylene blue in water, strip away the solvent environment and recompute the vertical excitation energies with TDDFT. This data set of energy gap fluctuations contains indirect solvent effects (ie. solvent-induced changes to chromophore geometry and motion) but no direct solvent effects (ie. solvent polarization effects or the effect of hydrogen bonding on the electronic excited state). Comparing resulting linear and nonlinear optical spectra to those computed for the same trajectory including the solvent environment, as well as the GBOM spectra, can help distinguish what type of effects are induced by direct solvent effects, indirect solvent effects and anharmonic vibrational motion of the chromophore not captured in the GBOM.

Fig. 4 shows the spectral density computed for the fully solvated MD trajectory and the stripped solvent trajectory, in comparison with GBOM spectral density. As can be seen, the direct solvent effects have a significant influence on the spectral density, and ignoring the condensed phase environment in computing the vertical excitation energies 


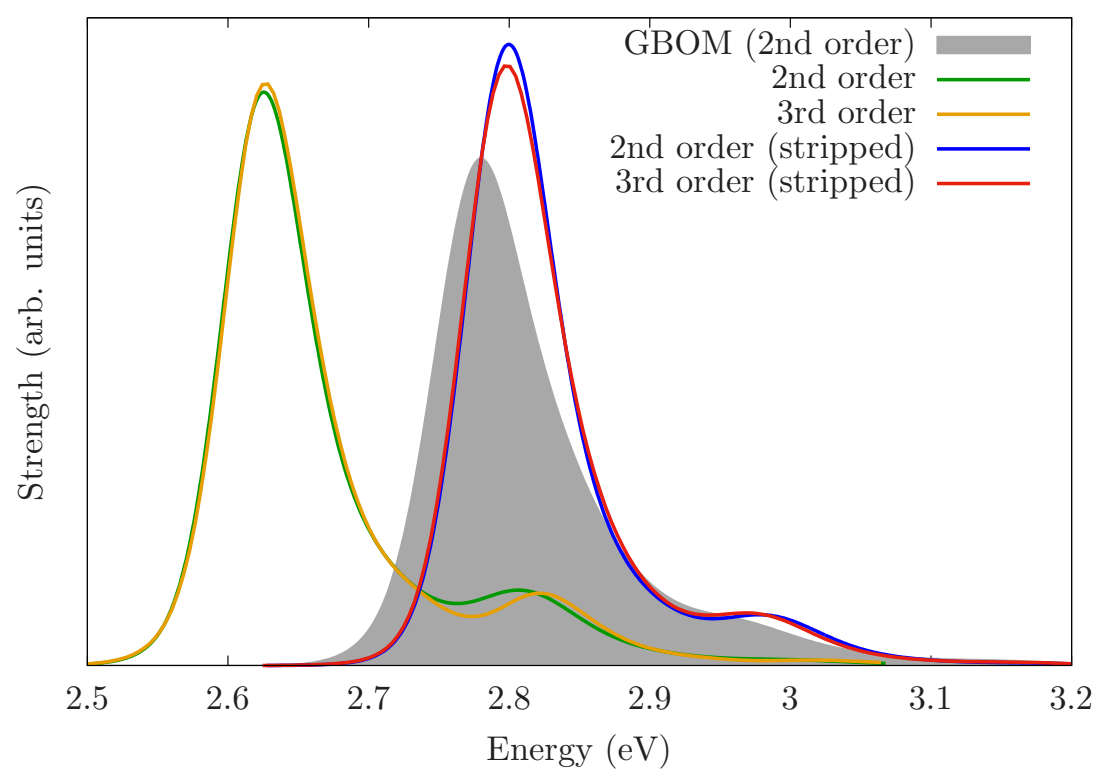

FIG. 5. Linear absorption spectrum computed in the cumulant approach for a single 8 ps MD trajectory of Methylene blue, once where the vertical excitation energies are computed within $6 \AA$ QM regions of explicit water molecules, and once where the solvent environment is stripped from all snapshots. The linear spectrum for the GBOM computed in the 2nd order cumulant approximation is shown in gray. For both the GBOM and the stripped solvent spectra, an effective solvent spectral density of the Debye form, with $\lambda_{\text {solv }}=0.011 \mathrm{eV}$ has been added to the system to produce spectra that are comparable in width with the fully solvated MD spectra.

leads to an underestimation in vibronic coupling to high frequency vibrational modes of the chromophore, as well as low frequency contributions to the spectral density. A similar effect has been observed recently for the spectral densities of anionic compounds in water, ${ }^{27}$ where the spectral density was also found to be very sensitive to the QM treatment of the condensed phase environment. As discussed in the main manuscript, the MD-based spectral densities in general show very similar features as the GBOM spectral density, with the main difference being intensities of specific high frequency vibrational modes and the fact that the GBOM spectral density lacks any low frequency contribution that we ascribe to coupled chromophore-environment collective motion. However, from Fig. 4 it is clear that that the vibronic peak positions predicted by the MD sampling of the PES are not exactly identical to the GBOM peaks, and some high frequency peaks in the spectral density beyond $1500 \mathrm{~cm}^{-1}$ appear red-shifted in the MD data. This effect can be ascribed to anharmonicities in the 
PES that are partially captured by the MD, but are missing from the GBOM. ${ }^{5}$

The discrepancies in the spectral density between stripped and fully solvated trajectories are reflected in the linear absorption spectra (see Fig. 5). Since the stripped trajectory lacks low frequency contributions to the spectral density due to solvent broadening, we add an effective solvent spectral density of the Debye form to its spectral density when computing linear spectra. The same parameters for the effective solvent environment are chosen as is done for the GBOM spectrum that is shown in comparison. Adding the effective solvent broadening through the solvent spectral density guarantees that the linear spectrum has a spectral width comparable to the fully solvated MD spectrum, while all direct solvent effects are still discarded. The stripped spectra appear blue-shifted by $\approx 0.2 \mathrm{eV}$ in comparison with the solvated MD spectra and are in much closer agreement with the 2nd order cumulant spectrum computed for the GBOM in vacuum, which we can ascribe to a lack of direct solvent polarization effects in both the GBOM and the stripped MD spectrum. More interestingly, the 2nd order cumulant linear spectra also show a change in shape when going from the fully solvated to the stripped spectra: The vibronic shoulder is more pronounced in the solvated spectra, which we can directly assign to the increased intensity in the high frequency $\left(\omega>1400 \mathrm{~cm}^{-1}\right.$ ) region of the spectral density (See Fig. 4). Furthermore, while the 3rd order cumulant correction for the stripped spectrum is very small, consisting of a slight redshift of the vibronic shoulder, the correction is much larger for the fully solvated spectrum and the vibronic shoulder is blueshifted instead, its peak moving further away from the 0-0 transition. The results for the linear spectrum suggest that at least some of the contribution to the 3rd order cumulant correction in the MD model is directly associated with direct solute-solvent interactions.

To further analyze the influence of direct solute-solvent coupling on nonlinear optical spectra, we compute time-series of integrated intensities for the two special points of the 2DES map discussed in the main manuscript (See Fig. 6 and 7). The special points correspond to the time evolution of the 0-0 transition (shown in black/gray), and the excited state relaxation from the 0-1 to the 0-0 transition (shown in magenta). In Fig. 6 integrated intensities of the two points on the 2DES map are shown for the GBOM, the solvated and the stripped MD trajectory as computed in the second order cumulant approximation. At this level of approximation, the effects of ignoring direct solute-solvent coupling are already visible. For the time evolution of the 0-0 transition, ignoring direct solute solvent coupling 


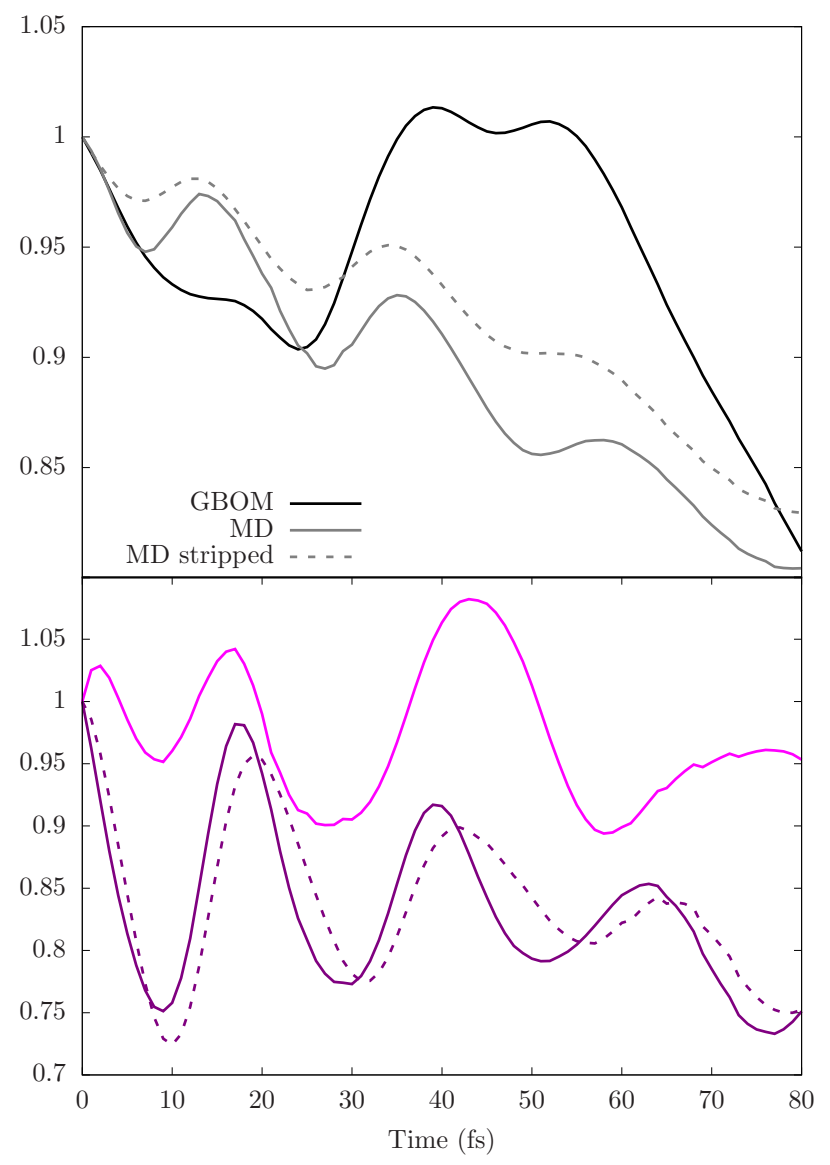

FIG. 6. Time evolution of integrated intensities of the 2DES maps computed in the 2nd order cumulant approach for a single 8 ps MD trajectory of Methylene blue, once where the vertical excitation energies are computed within $6 \AA$ QM regions of explicit water molecules, and once where the solvent environment is stripped from all snapshots, in comparison with the same data constructed for the GBOM. For both the GBOM and the stripped solvent spectra, an effective solvent spectral density of the Debye form, with $\lambda_{\text {solv }}=0.011 \mathrm{eV}$ has been added to the system. All integrated intensities are normalized by their values at $t=0$ fs to allow for an easier comparison.

leads to a slower overall decay of the signal, as well as a smaller period of oscillation for the oscillatory features. For the off diagonal vibronic cross peak, the overall decay of the signal is very similar between the stripped and the fully solvated MD and thus is not overly influenced by direct solute solvent coupling. However, there is a clearly visible increase in the period of oscillation for the oscillatory features of the stripped solvent trajectory. Thus removing the direct coupling between the solvent and the chromophore has a direct influence on specific vibronic peaks in the 2DES map within the second order cumulant approxima- 


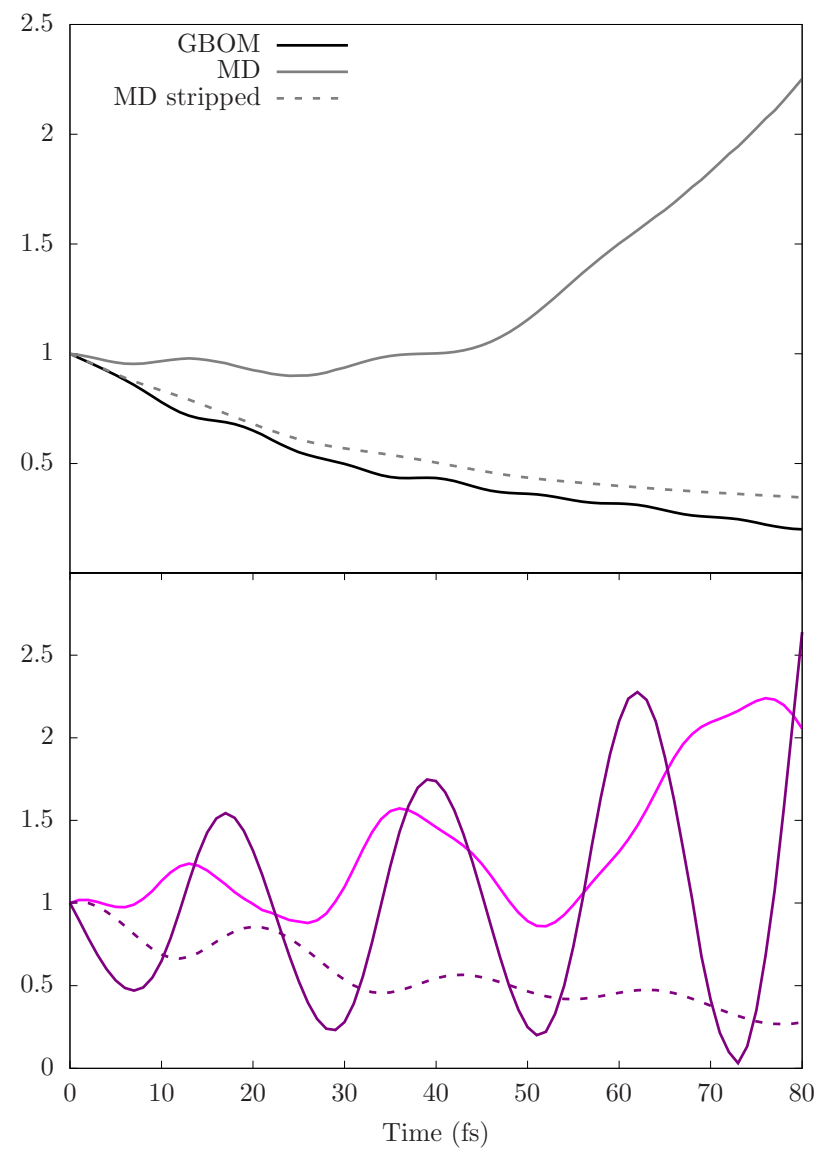

FIG. 7. Time evolution of integrated intensities of the 2DES maps computed in the 3rd order cumulant approach for a single 8 ps MD trajectory of Methylene blue, once where the vertical excitation energies are computed within $6 \AA$ QM regions of explicit water molecules, and once where the solvent environment is stripped from all snapshots, in comparison with the same data constructed for the GBOM. For both the GBOM and the stripped solvent spectra, an effective solvent spectral density of the Debye form, with $\lambda_{\text {solv }}=0.011 \mathrm{eV}$ has been added to the system. All integrated intensities are normalized by their values at $t=0$ fs to allow for an easier comparison.

tion, both for the overall decay of the signal which can be ascribed to a coupling to low frequency collective degrees of freedom and the fast oscillatory features which are due to high frequency vibrational modes of the chromophore.

Fig. 7 shows the same data as Fig. 6 but computed within the third order cumulant approximation rather than the second order cumulant approximation. We note that for the relaxation of the 0-0 transition, the fully solvated MD trajectory shows a divergent behavior. This can likely be ascribed to the short trajectory used, which is insufficient to fully converge 
the two-time correlation function of energy-gap fluctuations. However, it is also clear that the third order cumulant correction computed from the stripped solvent trajectory shows a very different behavior to the fully solvated trajectory, with oscillatory contributions to the off-diagonal vibronic peak being significantly underestimated in the stripped spectrum. It follows that a significant portion of the third order cumulant correction to the Methylene blue 2DES signals in the MD scheme is due to a direct coupling between the solute and the solvent environment, that cannot be captured in a GBOM model.

\section{QUANTIFYING THE AMOUNT OF NON-LINEAR COUPLING IN THE GBOM}

The third order cumulant approach outlined in this work is expected to work well for relatively small deviations from Gaussian energy gap fluctuations that are exactly described by the second order cumulant approximation. It is therefore interesting to quantify the degree of non-linearity in the coupling of the energy gap fluctuations to the nuclear degrees of freedom to obtain a measure for determining the reliability of the third order cumulant approximation. In the context of the GBOM, such a measure can be found in the difference between the classical and the quantum mechanical thermal average of energy gap fluctuations. Defining the difference between the two thermal averages as $\Delta \omega_{e g}^{\mathrm{av}, \mathrm{cl} / \mathrm{QM}}=\omega_{e g}^{\mathrm{av}, \mathrm{cl}}-\omega_{e g}^{\mathrm{av}, \mathrm{QM}}$ we find

$$
\Delta \omega_{e g}^{\mathrm{av}, \mathrm{cl} / \mathrm{QM}}=\frac{1}{2} \sum_{j} \frac{\Omega_{j j}^{2}}{\omega_{g, j}}\left[\frac{2}{\beta \omega_{g, j}}-\operatorname{coth}\left(\frac{\beta \omega_{g, j}}{2}\right)\right] .
$$

We note that the quantity $\Delta \omega_{e g}^{\text {av, cl/QM }}$ approaches 0 for $\beta \rightarrow 0$ or $\Omega_{j j} \rightarrow 0$. Eqn. 41 thus consists of a weighted sum of non-linear contributions to the energy gap fluctuations, with high frequency modes corresponding to $\beta \omega_{g, i}>>1$ being assigned more weight. $\Omega_{j j}$ approaches 0 only if $\mathbf{J}=\mathbf{I}$ and $\omega_{g, i}=\omega_{e, i}$, and thus $\Delta \omega_{e g}^{\text {av, cl/QM }}$ serves as a simple, physically motivated measure of non-Gaussian fluctuations introduced through both the Duschinsky mode mixing and the change in curvature between ground and excited state PESs. Furthermore, the sign of $\Delta \omega_{e g}^{\mathrm{av}, \mathrm{cl} / \mathrm{QM}}$ is determined by the sign of the $\Omega_{j j}^{2}$ terms in the weighted sum. If $\Delta \omega_{e g}^{\mathrm{av}, \mathrm{cl} / \mathrm{QM}}$ is positive this can be interpreted as the excited state PES having on average a smaller curvature than the ground state PES for the high frequency modes significantly contributing to the weighted sum. 


\begin{tabular}{c|c|c|c|c} 
& 2-mode model & Nile red & Nile red $(\mathbf{J}=\mathbf{I})$ & Methylene blue \\
\hline$\Delta \omega_{e g}^{\text {av, cl/QM }}$ & 0.0011 & 0.0349 & 0.0363 & 0.0777
\end{tabular}

TABLE I. Values of $\Delta \omega_{e g}^{\mathrm{av}, \mathrm{cl} / \mathrm{QM}}$ in $\mathrm{eV}$ for the GBOM systems reported in the main manuscript.

Values of $\Delta \omega_{e g}^{\mathrm{av}, \mathrm{cl} / \mathrm{QM}}$ for the systems considered in the main text can be found in Table I.

All the systems considered in this work have $\Delta \omega_{e g}^{\text {av, cl/QM }}>0$, suggesting that on average the curvature of the excited state PES is less than that of the ground state PES. Thus for the systems considered in this work the average energy gap is always overestimated by the classical sampling of nuclear degrees of freedom. From Table I it also becomes clear that by the measure defined here, the GBOM for Methylene blue has the most non-linear coupling of the systems considered in this work, suggesting that a low order cumulant approximation might be less successful in this system than for Nile red.

\section{REFERENCES}

${ }^{1}$ S. Mukamel, Principles of Nonlinear Optical Spectroscopy (Oxford University Press, New York, 1995).

${ }^{2}$ E. Condon, "A Theory of Intensity Distribution in Band Systems," Phys. Rev. 28, 11821201 (1926).

${ }^{3}$ E. Condon, "Nuclear Motion Associated with Electron Transitions in Diatomic molecles," Phys. Rev. 32, 858-872 (1928).

${ }^{4}$ A. F. Fidler and G. S. Engel, "Nonlinear spectroscopy theory of displaced harmonic oscillators with differing curvatures: A correlation function approach," J. Phys. Chem. A 117, 9444-9453 (2013).

${ }^{5}$ T. J. Zuehlsdorff, A. Montoya-Castillo, J. A. Napoli, T. E. Markland, and C. M. Isborn, "Optical spectra in the condensed phase: Capturing anharmonic and vibronic features using dynamic and static approaches," J. Chem. Phys 151, 074111 (2019).

${ }^{6}$ J. S. Bader and B. J. Berne, "Quantum and classical relaxation rates from classical simulations," J. Chem. Phys. 100, 8359 (1994).

${ }^{7}$ H. Kim and P. J. Rossky, "Evaluation of Quantum Correlation Functions from Classical Data," J. Phys. Chem. B 106, 8240 (2002). 
${ }^{8}$ S. A. Egorov, K. F. Everitt, and J. L. Skinner, "Quantum Dynamics and Vibrational Relaxation," J. Phys. Chem. A 103, 9494-9499 (1999).

${ }^{9}$ I. R. Craig and D. E. Manolopoulos, "Quantum statistics and classical mechanics: Real time correlation functions from ring polymer molecular dynamics," J. Chem. Phys. 121, 3368 (2004).

${ }^{10}$ R. Ramirez, T. Lopez-Ciudad, P. K. P, and D. Marx, "Quantum corrections to classical time-correlation functions: Hydrogen bonding and anharmonic floppy modes," J. Chem. Phys. 121, 3973 (2004).

${ }^{11}$ K. A. Jung, P. E. Videla, and V. S. Batista, "Inclusion of nuclear quantum effects for simulations of nonlinear spectroscopy," J. Chem. Phys. 148, 244105 (2018).

${ }^{12}$ M. J. Frisch, G. W. Trucks, H. B. Schlegel, G. E. Scuseria, M. A. Robb, J. R. Cheeseman, G. Scalmani, V. Barone, G. A. Petersson, H. Nakatsuji, X. Li, M. Caricato, A. V. Marenich, J. Bloino, B. G. Janesko, R. Gomperts, B. Mennucci, H. P. Hratchian, J. V. Ortiz, A. F. Izmaylov, J. L. Sonnenberg, D. Williams-Young, F. Ding, F. Lipparini, F. Egidi, J. Goings, B. Peng, A. Petrone, T. Henderson, D. Ranasinghe, V. G. Zakrzewski, J. Gao, N. Rega, G. Zheng, W. Liang, M. Hada, M. Ehara, K. Toyota, R. Fukuda, J. Hasegawa, M. Ishida, T. Nakajima, Y. Honda, O. Kitao, H. Nakai, T. Vreven, K. Throssell, J. A. Montgomery, Jr., J. E. Peralta, F. Ogliaro, M. J. Bearpark, J. J. Heyd, E. N. Brothers, K. N. Kudin, V. N. Staroverov, T. A. Keith, R. Kobayashi, J. Normand, K. Raghavachari, A. P. Rendell, J. C. Burant, S. S. Iyengar, J. Tomasi, M. Cossi, J. M. Millam, M. Klene, C. Adamo, R. Cammi, J. W. Ochterski, R. L. Martin, K. Morokuma, O. Farkas, J. B. Foresman, and D. J. Fox, "Gaussian Development Version, Revision I.09," (2016), Gaussian Inc. Wallingford CT.

${ }^{13}$ T. Yanai, D. P. Tew, and N. C. Handy, "A new hybrid exchange-correlation functional using the Coulomb-attenuating method (CAM-B3LYP)," Chem. Phys. Lett. 393, 51-57 (2004).

${ }^{14}$ T. H. Dunning Jr., "Gaussian basis sets for use in correlated molecular calculations. i. the atoms boron through neon and hydrogen," J. Chem. Phys. 90, 1007 (1989).

${ }^{15}$ R. Cammi, S. Corni, B. Mennucci, and J. Tomasi, "Electronic excitation energies of molecules in solution: State specific and linear response methods for nonequilibrium continuum solvation models," J. Chem. Phys. 122, 104513 (2005).

${ }^{16} \mathrm{~J}$. Tomasi, B. Mennucci, and R. Cammi, "Quantum mechanical continuum solvation models," Chem. Rev. 105, 2999-3094 (2005), pMID: 16092826. 
${ }^{17}$ S. Hirata and M. Head-Gordon, "Time-dependent density functional theory within the Tamm-Dancoff approximation," Chem. Phys. Lett. 314, 291-299 (1999).

${ }^{18}$ F. Santoro, A. Lami, R. Improta, J. Bloino, and V. Barone, "Effective method for the computation of optical spectra of large molecules at finite temperature including the duschinsky and herzberg-teller effect: The qx band of porphyrin as a case study," J. Chem. Phys. 128, 224311 (2008).

${ }^{19}$ A. Baiardi, J. Bloino, and V. Barone, "General time dependent approach to vibronic spectroscopy including franck-condon, herzberg-teller, and duschinky effects," J. Chem. Theory Comput. 9, 4097-4115 (2013).

${ }^{20}$ B. de Souza, F. Neese, and R. Izsák, "On the theoretical prediction of fluorescence rates from first principles using the path integral approach," J. Chem. Phys. 148, 034104 (2018).

${ }^{21}$ M. M. Davis and H. B. Helzer, "Titrimetric and equilibrium studies using indicators related to nile blue a." Anal. Chem. 38, 451-461 (1966), https://doi.org/10.1021/ac60235a020.

${ }^{22}$ P. Eastman, J. Swails, J. D. Chodera, R. T. McGibbon, Y. Zhao, K. A. Beauchamp, L.-P. Wang, A. C. Simmonett, M. P. Harrigan, C. D. Stern, R. P. Wiewiora, B. R. Brooks, and V. S. Pande, "Openmm 7: Rapid development of high performance algorithms for molecular dynamics," PLOS Comput. Biol. 13, 1-17 (2017).

${ }^{23}$ W. L. Jorgensen, J. Chandrasekhar, and J. D. Madura, "Comparison of simple potential functions for simulating liquid water," J. Chem. Phys. 79, 926 (1983).

${ }^{24}$ J. T. Horton, A. E. A. Allen, L. S. Dodda, and D. J. Cole, "Qubekit: Automating the derivation of force field parameters from quantum mechanics," J. Chem. Inf. Model 59, 1366-1381 (2019), pMID: 30742438, https://doi.org/10.1021/acs.jcim.8b00767.

${ }^{25}$ I. S. Ufimtsev and T. J. Martinez, "Quantum Chemistry on Graphical Processing Units. 3. Analytical Energy Gradients and First Principles Molecular Dynamics," J. Chem. Theory Comput. 5, 2619-2628 (2009).

${ }^{26}$ C. M. Isborn, N. Luehr, I. S. Ufimtsev, and T. J. Martínez, "Excited-State Electronic Structure with Configuration Interaction Singles and Tamm-Dancoff Time-Dependent Density Fucntional Theory on Graphical Processing Units," J. Chem. Theory Comput. 7, 1814-1823 (2011).

${ }^{27}$ T. J. Zuehlsdorff, H. Hong, L. Shi, and C. M. Isborn, "Influence of Electronic Polarization on the Spectral Density," J. Phys. Chem. B 124, 531-543 (2020). 
